TITLE:

\title{
Frequency response-based damage identification by minimum constitutive relation error and sparse regularization
}

\section{$\operatorname{AUTHOR}(\mathrm{S})$ :}

Guo, Jia; Wang, Li; Takewaki, Izuru

\section{CITATION:}

Guo, Jia ... [et al]. Frequency response-based damage identification by minimum constitutive relation error and sparse regularization. Journal of Sound and Vibration 2019, 443: $270-292$

ISSUE DATE:

2019-03-17

URL:

http://hdl.handle.net/2433/244214

\section{RIGHT:}

(c) 2019. This manuscript version is made available under the CC-BY-NC-ND 4.0 license

http://creativecommons.org/licenses/by-nc-nd/4.0/:; The full-text file will be made open to the public on 17 March 2021 in accordance with publisher's 'Terms and Conditions for Self-Archiving'.; この論文は出版社版でありません。引用の際 には出版社版をご確認ご利用ください。; This is not the published version. Please cite only the published version. 


\title{
Frequency response-based damage identification in frames by minimum constitutive relation error and sparse regularization
}

\author{
Jia Guo ${ }^{\mathrm{a}}$, Li Wang ${ }^{\mathrm{b}, *}$, Izuru Takewaki ${ }^{\mathrm{a}}$ \\ ${ }^{a}$ Department of architecture and architectural engineering, Kyoto University, Kyoto, Japan \\ ${ }^{b}$ Department of applied mechanics and engineering, Sun Yat-sen University, Guangzhou, \\ P.R. China
}

\begin{abstract}
The objective of this paper is to provide a new damage identification method using frequency response data. In this approach, the inverse identification problem is treated as a nonlinear optimization problem whose objective function is just the constitutive relation error (CRE). To circumvent the ill-posedness of the inverse problem which is caused by use of the possibly insufficient data and enhance the robustness of the identification process, the sparse regularization is introduced where the $\ell_{1}$-norm regularization term is added to the original CRE function. In regard to the minimum solution of the sparse-regularized CRE objective function, the alternating minimization (AM) method is established. The attractive features of the present damage identification approach are: (a) while coping with the sparse regularization, a closed-form solution is obtained due to the decoupling of the CRE function with respect to the damage parameters and hence the sparse regularization term would introduce little computational complexity; (b) the sparse regularization parameters are directly determined by a simple threshold setting method; (c) no sensitivity analysis is involved herein. Numerical examples are conducted to verify the proposed approach and the results show that the sparse regularization obviously improves the accuracy and robustness for the identified damages.
\end{abstract}

\footnotetext{
* Corresponding author

Email address: wangli75@mail.sysu.edu.cn (Li Wang)
} 
Keywords: damage identification, constitutive relation error, sparse regularization, frequency response data, alternating minimization method

\section{Introduction}

Academic research regarding vibration-based damage identification has been rapidly expanded over the past decades $[1,2,3,4]$. The methods, using dynamic response data in the damage identification process, could be classified into two groups: methods based on signals and methods based on models [5]. Signalbased methods received much attention in recent years and have been successfully applied to detect and locate structural damages $[6,7]$. They are typically non-parametric methods and able to avoid modeling errors and high computational costs during numerical simulations. However, these methods are hard to assess the damage severity, sensitive to the measurement noise and mostly limited up to small-sized structures[5]. In contrast, the advantage of the methods based on models is obvious: able to identify the damage location and severity simultaneously and capable to take into account all of the available structural information.

Classically, the model-based methods treat damage identification as a constrained nonlinear optimization problem with the structural model parameters such as mass, stiffness and damping forming the basic variables. The objective function of the nonlinear problem is usually set up by measuring the discrepancies between the numerical model and the dynamic test results. The selection of the objective function is a crucial issue. It not only affects the interpretation of the best correlation, but also influences the behavior of the utilized optimization algorithm[8]. Weighted least squares of the errors between the measured data and the derived data is generally chosen as the objective function[9]. To solve this optimization problem, the genetic algorithms (GA) and the sensitivitybased approaches are commonly used[10, 11, 12]. Other opimization methods include the minimization of rank perturbation theory[13] and the 'residual force' $\operatorname{method}[14]$. 
Recently, usage of the error in constitutive relation has caught the attention of researchers. This idea was first proposed for model updating analysis and then extended to structural damage identification problems in linear statics[15], transient dynamics[16], elastoplastics[17] and so on. In this approach, the objective function is defined as the constitutive relation error (CRE), that is, an energy inner product of the residual of the constitutive equation connecting the admissible stresses and the kinematically admissible displacements. It is remarkable that the CRE objective function is separately convex, which guarantees the appropriate application of the efficient alternating minimization (AM) approach $[18,19]$, to get the solution of this nonlinear optimization problem. To this end, the sensitivity analysis is no longer needed.

For large scale civil structures, it is often required to identify the structural parameters based on the incomplete structural vibration response measurement data. This is essentially an ill-posed inverse problem which is caused by the possibly insufficient amount of the measurement data. Attempting to circumvent this drawback, regularization techniques such as the Tikhonov regularization and the sparse regularization are always introduced. The idea that all the damages or changes of parameters are as small as possible is implicated in the Tikhonov regularization[20]. Such an idea is reasonable for model updating however, it may not be appropriate for damage identification since large damages may occur. On the other hand, damages always occur at a few locations where maximum-stress/plasticity appears, impact loads work or crack propagates for practical structures. This would be appropriately implicated in the sparse regularization[21, 22], which weakly enforces the sparsity constraint for the damage locations.

In this paper, a new structural damage identification is proposed for the incomplete measured frequency response data using the minimum constitutive relation error (min-CRE) approach and the sparse regularization. The first objective of this paper is to clarify the mathematical advantages of the decoupled CRE objective function with respect to the damage parameters. In fact, the sparse regularization has already been incorporated into the sensitivity-based 
approaches[23]. However, it is noteworthy that a linear least-squares function along with the prescribed sparse regularization is a non-linear and non-quadratic function and its minimization shall be obtained through iterative algorithms, which is costly compared to the case with the Tikhonov regularization. In general, the sparse regularization problem requires significantly greater computational effort and no closed-form solution exists. There are several families of computational methods to get sparse solutions: greedy pursuit, convex relaxation, non-convex optimization, Bayesian framework and brute force[22]. In this research, however, the inherent decoupling of the CRE function with respect to the damage parameters makes every parameter-update-step solved as a series of single-variable optimization problem and hence results in a closed-form solution for the sparse regularization problem. This introduces little additionally computational effort caused by the sparse regularization.

The second objective of this paper is to present a novel and simple threshold setting method to properly determine the sparse regularization parameters. There are several ways to obtain the general optimal regularization parameters for inverse problems in mathematics: discrepancy principle (DP)[24], ordinary and generalized cross validations (GCV)[25], L-curve criterion[26] and so on. However, the selection criterion for the sparse regularization is very limited because the problem generally has no closed-form solution. An appropriate regularization parameter is typically selected by experience[27, 28]. The reference[29] proposed two possible strategies of selecting the sparse regularization parameter: the first selection method utilizes the residual and solution norms to determine the appropriate range of the regularization parameter while the other is developed based on the DP. In both strategies, the linear leastsquares problems under several trial sparse regularization parameters should be solved and this obviously leads to prohibitively high computation cost. In this paper, the determination of the regularization parameter would be straightforward and require no repeated computation by the proposed threshold setting method.

In this research, the frequency response function (FRF) and natural frequen- 
cies data, directly extracted from the dynamic test, are used as the measured data. Vibration-based damage identification using FRF measurements has been studied by a number of references[30, 31] and it has been proved that FRF data are more reliable and practical to provide abundant information at measured degrees of freedom and at a great number of desired frequencies[32] without being contaminated by any modal extraction errors and loss of information. Particular attention should be paid to properly select the frequency ranges of the FRF data. The remainder of this paper is organized as follows. The basic inverse identification problem by the min-CRE principle is introduced in Section 2. In Section 3, the sparse regularization is applied to enhance the CRE objective function and then, an AM method is applied to solve this nonlinear optimization problem. Numerical tests are performed in Section 4 and final conclusions are drawn in Section 5.

\section{Problem statement}

\subsection{The baseline model}

Consider an elastic structure occupying the open domain $\Omega$ and bounded by the boundary $\partial \Omega$. The vibration of this linear elastic problem in frequency domain is divided into three sets of differential equations for the displacement field $\boldsymbol{u}$ and the stress field $\boldsymbol{\sigma}$.

\section{- Kinematic constraints}

$$
\boldsymbol{u} \in \mathcal{U}:=\left\{\begin{array}{l}
\boldsymbol{\varepsilon}=\mathcal{S} \boldsymbol{u} \text { in } \Omega \\
\boldsymbol{u}=\boldsymbol{u}_{p} \text { over } \partial \Omega_{u}
\end{array}\right.
$$

where $\mathcal{S}$ is a suitable linear differential operator and $\boldsymbol{u}_{p}$ is the prescribed value of the displacement filed $\boldsymbol{u}$ on the Dirichlet boundary $\partial \Omega_{u} \cdot \varepsilon$ represents the deformation tensor.

\section{- Equilibrium equations}

$$
\boldsymbol{\sigma} \in \Im:=\left\{\begin{array}{l}
\mathcal{L} \boldsymbol{\sigma}+\boldsymbol{b}=\boldsymbol{\tau} \text { in } \Omega \\
\boldsymbol{\sigma} \cdot \boldsymbol{n}=\boldsymbol{t} \text { over } \partial \Omega_{f}
\end{array}\right.
$$


where $\mathcal{L}$ is the divergence operator on stress filed $\boldsymbol{\sigma}$ and $n$ the external unit vector normal to the Neumann boundary $\partial \Omega_{f}$. Over $\partial \Omega_{f}$, the traction field $\boldsymbol{t}$ is prescribed. In addition, $\boldsymbol{b}$ denotes the body force while $\boldsymbol{\tau}$ the inertial force.

\section{- Constitutive relations}

$$
\left\{\begin{array}{l}
\boldsymbol{\sigma}=\mathbb{D}: \boldsymbol{\varepsilon} \text {-Hooke's law, } \\
\boldsymbol{\tau}=-\omega^{2} \rho \boldsymbol{u} \text {-Newton's law. }
\end{array}\right.
$$

where $\mathbb{D} \in \mathcal{C}$ is an elastic modulus tensor with appropriate material properties and $\rho$ is the density which is assumed to be constant in this research. It should be noted that only the case of forced vibrations problems are considered and the equations are written in the frequency domain with $\omega$ being the angular

\subsection{CRE function for a given frequency}

To define the constitutive relation error (CRE), consider an admissible pair $(\boldsymbol{u}, \boldsymbol{\sigma})$ which satisfies the kinematic constrains (equation (1)) and the equilibrium equations (equation (2)) respectively. It is easily known that, if the conelastic modulus tensor $\mathbb{D}$, the above admissible solution trio $(\boldsymbol{u}, \boldsymbol{\sigma}, \mathbb{D})$ would be the exact solution $\left(\boldsymbol{u}_{\mathrm{ex}}, \boldsymbol{\sigma}_{\mathrm{ex}}, \mathbb{D}_{\mathrm{ex}}\right)$ of this dynamic linear elastic problem.

As a result, to measure the distance from the admissible solution to the exact solution in the energy product, the CRE for a given frequency is expressed as

$$
\begin{aligned}
e_{C R E}(\boldsymbol{u}, \boldsymbol{\sigma}, \mathbb{D})=\frac{1}{2} \int_{\Omega}\left\{r_{1} \operatorname{Tr}\left[(\boldsymbol{\sigma}-\mathbb{D}: \boldsymbol{\varepsilon}(\boldsymbol{u})): \mathbb{D}^{-1}:(\boldsymbol{\sigma}-\mathbb{D}: \boldsymbol{\varepsilon}(\boldsymbol{u}))\right]+\right. \\
\left.r_{2}\left(\rho \omega^{2}\right)^{-1}\left[\left(\boldsymbol{\tau}(\boldsymbol{\sigma})+\rho \omega^{2} \boldsymbol{u}\right):\left(\boldsymbol{\tau}(\boldsymbol{\sigma})+\rho \omega^{2} \boldsymbol{u}\right)\right]\right\} d \Omega
\end{aligned}
$$

where $r_{1}, r_{2}$ are the corresponding weight coefficients $\left(r_{1}+r_{2}=1\right)$. The values of these coefficients depend on the relative reliability between the constitutive relations based on the Hooke's law and based on the Newton's law and are fixed to be $r_{1}=r_{2}=0.5$ in this work. 
Further considering the kinematic constrains and the equilibrium equations, the CRE in equation (4) could be rewritten as

$$
\begin{aligned}
e_{C R E}(\boldsymbol{u}, \boldsymbol{\sigma}, \mathbb{D})=\frac{1}{2} \int_{\Omega}\left\{r_{1} \operatorname{Tr}\left[(\boldsymbol{\sigma}-\mathbb{D}: \mathcal{S} \boldsymbol{u}): \mathbb{D}^{-1}:(\boldsymbol{\sigma}-\mathbb{D}: \mathcal{S} \boldsymbol{u})\right]+\right. \\
\left.r_{2}\left(\rho \omega^{2}\right)^{-1}\left[\left(\mathcal{L} \boldsymbol{\sigma}+\boldsymbol{b}+\rho \omega^{2} \boldsymbol{u}\right):\left(\mathcal{L} \boldsymbol{\sigma}+\boldsymbol{b}+\rho \omega^{2} \boldsymbol{u}\right)\right]\right\} d \Omega
\end{aligned}
$$

For the structure which is of interest in a frequency range $\left[\omega_{\min }, \omega_{\max }\right]$, the frequency weighting factor $z(\omega)$ is introduced

$$
\int_{\omega_{\min }}^{\omega_{\max }} z(\omega)=1, z(\omega)>0
$$

Then the total CRE in the given frequency range is established as

$$
F(\boldsymbol{u}, \boldsymbol{\sigma}, \mathbb{D}):=\int_{\omega_{\min }}^{\omega_{\max }} e_{C R E}(\boldsymbol{u}, \boldsymbol{\sigma}, \mathbb{D}) z(\omega) d \omega
$$

Based on the equations above, the damage identification problem is formulated as

$$
\begin{aligned}
& \arg \min F(\boldsymbol{u}, \boldsymbol{\sigma}, \mathbb{D}) \\
& \text { subject to } \boldsymbol{u} \in \mathcal{U}, \boldsymbol{\sigma} \in \Im, \mathbb{D} \in \mathcal{C}, \boldsymbol{u}=\hat{\boldsymbol{u}}_{k}, k \in \wp
\end{aligned}
$$

where $\left\{\hat{\boldsymbol{u}}_{k}, k \in \wp\right\}$ is the incomplete frequency response data measured by finite sensors on the set of points $\{k \in \wp\}$. Equation (8) is known as the min-CRE principle for inverse identification problems $[33,34]$ and $F(\boldsymbol{u}, \boldsymbol{\sigma}, \mathbb{D})$ is known as the CRE objective function. For each frequency $\omega_{i}, z\left(\omega_{i}\right)$ is taken equally herein. The CRE function is chosen as the objective function in this research due to the following crucial features,

- Separate convexity: the separately convex property of the proposed objective function is of critical importance for the application of the alternating minimization(AM) method because only then, each alternating step become well-posed[35]. (Refer to section 3.1)

- Decoupling with respect to the damage parameters: under the constant stiffness assumption in every element, the CRE objective function is decoupled with respect to the damage parameters, or specifically, minimization 
of the CRE objective function over the damage parameters gives rise to a series of single-variable minimization problems. With the decoupled objective function, the sparse regularization, which is to be introduced later, will be easily tackled by the closed-form solution with little additional computational efforts. (Refer to section 3.3)

In the following, the inverse identification problem is solved in a finite element environment which requires to discretize the CRE objective function at first. A typical finite element, $e$, is defined by local nodes $i, j, k, \ldots$ and for each frequency, the displacement $\boldsymbol{u}$ and stress $\boldsymbol{\sigma}$ and the trial counterparts $\delta \boldsymbol{u}$ and $\delta \boldsymbol{\sigma}$ within the element $e$ is approximated as

$$
\left\{\begin{array}{l}
\boldsymbol{u} \approx \boldsymbol{N}_{1} \tilde{\boldsymbol{u}}^{e}, \delta u \approx \boldsymbol{N}_{1} \delta \tilde{\boldsymbol{u}}^{e} \\
\boldsymbol{\sigma} \approx \boldsymbol{N}_{2} \tilde{\boldsymbol{q}}^{e}=\boldsymbol{N}_{2} \tilde{\boldsymbol{q}}_{0}^{e}+\boldsymbol{N}_{2} \tilde{\boldsymbol{q}}_{p}^{e}, \delta \boldsymbol{\sigma} \approx \boldsymbol{N}_{2} \delta \tilde{\boldsymbol{q}}^{e}=\boldsymbol{N}_{2} \delta \tilde{\boldsymbol{q}}_{0}^{e}
\end{array}\right.
$$

where $\boldsymbol{N}_{1}$ and $\boldsymbol{N}_{2}$ are called shape functions (or interpolation functions) corresponding to nodal displacement vector $\tilde{\boldsymbol{u}}^{e}$ and nodal force vector $\tilde{\boldsymbol{q}}^{e}$ separately. The nodal force $\tilde{\boldsymbol{q}}^{e}$, obtained from a mathematical integration of stress $\boldsymbol{\sigma}$, is dynamically equivalent to the addition of the boundary forces $\tilde{\boldsymbol{q}}_{p}^{e}$ and the remaining homogeneous forces $\tilde{\boldsymbol{q}}_{0}^{e}$. As is noteworthy, the inhomogeneous part $\boldsymbol{N}_{2}^{p} \tilde{\boldsymbol{q}}_{p}^{e}$ vanish in the virtual stress $\delta \boldsymbol{\sigma}$. Additionally, the nodal force $\tilde{\boldsymbol{q}}^{e}$ should contain the same number of components as the corresponding nodal displacement $\tilde{\boldsymbol{u}}^{e}$ and be ordered in the appropriate corresponding directions. For three-dimensional beam elements, details on the expressions for the nodal displacement vector $\tilde{\boldsymbol{u}}^{e}$ and the nodal force vector $\tilde{\boldsymbol{q}}^{e}$ and the derivation for the shape functions $\boldsymbol{N}_{1}$ and $\boldsymbol{N}_{2}$ are given in Appendix.

Using equations (1) and (2), one could also obtain the following expression.

$$
\left\{\begin{array}{l}
\boldsymbol{\varepsilon}=\mathcal{S} \boldsymbol{u} \approx \mathcal{S} \boldsymbol{N}_{1} \tilde{\boldsymbol{u}}^{e}=\boldsymbol{B}_{1} \tilde{\boldsymbol{u}}^{e} \\
\mathcal{L} \boldsymbol{\sigma} \approx \mathcal{L} \boldsymbol{N}_{2} \tilde{\boldsymbol{q}}^{e}=\boldsymbol{B}_{2} \tilde{\boldsymbol{q}}^{e}
\end{array}\right.
$$

With these preparations and further considering the CRE in equation (5), one 
can have the discrete form of the CRE as follows.

$$
\begin{aligned}
e_{C R E}(\tilde{\boldsymbol{u}}, \tilde{\boldsymbol{q}}, \boldsymbol{D}) & =\frac{1}{2} \int_{\Omega}\left\{r_{1}\left(\boldsymbol{N}_{2} \tilde{\boldsymbol{q}}-\boldsymbol{D} \boldsymbol{B}_{1} \tilde{\boldsymbol{u}}\right)^{T} \boldsymbol{D}^{-1}\left(\boldsymbol{N}_{2} \tilde{\boldsymbol{q}}-\boldsymbol{D} \boldsymbol{B}_{1} \tilde{\boldsymbol{u}}\right)+\right. \\
& \left.r_{2}\left(\rho \omega^{2}\right)^{-1}\left(\boldsymbol{B}_{2} \tilde{\boldsymbol{q}}+\boldsymbol{b}+\rho \omega^{2} \boldsymbol{N}_{1} \tilde{\boldsymbol{u}}\right)^{T}\left(\boldsymbol{B}_{2} \tilde{\boldsymbol{q}}+\boldsymbol{b}+\rho \omega^{2} \boldsymbol{N}_{1} \tilde{\boldsymbol{u}}\right)\right\} d \Omega
\end{aligned}
$$

As for damage identification, the damage parameters $\tilde{\boldsymbol{\theta}}=\left[\theta_{0}:=1 ; \theta_{1} ; \ldots ; \theta_{N}\right], 0 \leq$ $\theta_{e} \leq 1, e=1,2, \ldots, N$ with $\theta_{e}=1$ meaning no damage of the $e$ th element are assumed to be linearly implicated in the elastic matrix $\boldsymbol{D}$, or in other words, the elastic matrix $\boldsymbol{D}$ is an affine function of the damage parameters $\theta$, i.e., $\boldsymbol{D}=\boldsymbol{D}(\tilde{\boldsymbol{\theta}}):=\theta_{e} \boldsymbol{D}_{e}$ for element $e$ where $\boldsymbol{D}_{e}$ is termed the elemental elastic matrix of the intact structure. Obviously, for an intact structure, there is $\tilde{\boldsymbol{\theta}}=\tilde{\boldsymbol{\theta}}_{0}:=[1 ; 1 ; \ldots ; 1]$. As a result, equation(11) can be equally described as

$$
\begin{aligned}
e_{C R E}(\tilde{\boldsymbol{u}}, \tilde{\boldsymbol{q}}, \tilde{\boldsymbol{\theta}}) & =\frac{1}{2} \sum_{e=1}^{N} \int_{\Omega^{e}}\left\{r_{1}\left(\boldsymbol{N}_{2} \tilde{\boldsymbol{q}}^{e}-\theta_{e} \boldsymbol{D}_{e} \boldsymbol{B}_{1} \tilde{\boldsymbol{u}}^{e}\right)^{T}\left(\theta_{e} \boldsymbol{D}_{e}\right)^{-1}\right. \\
& \left(\boldsymbol{N}_{2} \tilde{\boldsymbol{q}}^{e}-\theta_{e} \boldsymbol{D}_{e} \boldsymbol{B}_{1} \tilde{\boldsymbol{u}}^{e}\right)+r_{2}\left(\rho \omega^{2}\right)^{-1}\left(\boldsymbol{B}_{2} \tilde{\boldsymbol{q}}^{e}+\boldsymbol{b}+\rho \omega^{2} \boldsymbol{N}_{1} \tilde{\boldsymbol{u}}^{e}\right)^{T} \\
& \left.\left(\boldsymbol{B}_{2} \tilde{\boldsymbol{q}}^{e}+\boldsymbol{b}+\rho \omega^{2} \boldsymbol{N}_{1} \tilde{\boldsymbol{u}}^{e}\right)\right\} d \Omega
\end{aligned}
$$

With these notification, it is obvious that the CRE objective function (12) is decoupled with respect to the damage parameters $\tilde{\boldsymbol{\theta}}$, i.e., in every single equation of the equation (12), only one of the parameters $\tilde{\boldsymbol{\theta}}$ arises. Such a decoupling will act as a particular role in later analysis when the sparse regularization is considered.

\subsection{Sparse regularization}

In practice, the amount of the measured data is always limited and this may make the damage identification problem ill-posed and very sensitive to the measurement noise. To circumvent the ill-posedness and improve the robustness, the sparse regularization technique is reasonably introduced. The main prerequisite is that the change of damage parameters $\tilde{\boldsymbol{\theta}}-\tilde{\boldsymbol{\theta}}_{0}$ must be sparse and no other information regarding the damage is required. By incorporating the sparse regularization into the objective function, the damage identification 
problem is reformulated as

$$
\begin{aligned}
e_{C R E}(\tilde{\boldsymbol{u}}, \tilde{\boldsymbol{q}}, \tilde{\boldsymbol{\theta}})= & \frac{1}{2} \int_{\Omega}\left\{r_{1}\left(\boldsymbol{N}_{2} \tilde{\boldsymbol{q}}-\boldsymbol{D}(\tilde{\boldsymbol{\theta}}) \boldsymbol{B}_{1} \tilde{\boldsymbol{u}}\right)^{T} \boldsymbol{D}(\tilde{\boldsymbol{\theta}})^{-1}\left(\boldsymbol{N}_{2} \tilde{\boldsymbol{q}}-\boldsymbol{D}(\tilde{\boldsymbol{\theta}}) \boldsymbol{B}_{1} \tilde{\boldsymbol{u}}\right)+\right. \\
& \left.r_{2}\left(\rho \omega^{2}\right)^{-1}\left(\boldsymbol{B}_{2} \tilde{\boldsymbol{q}}+\boldsymbol{b}+\rho \omega^{2} \boldsymbol{N}_{1} \tilde{\boldsymbol{u}}\right)^{T}\left(\boldsymbol{B}_{2} \tilde{\boldsymbol{q}}+\boldsymbol{b}+\rho \omega^{2} \boldsymbol{N}_{1} \tilde{\boldsymbol{u}}\right)\right\} d \Omega \\
& \lambda\left\|\boldsymbol{\Delta}\left(\tilde{\boldsymbol{\theta}}-\tilde{\boldsymbol{\theta}}_{0}\right)\right\|_{1}^{1}
\end{aligned}
$$

where $\boldsymbol{\Delta}=\operatorname{diag}\left(\left[\Delta_{1}, \ldots, \Delta_{N}\right]\right)$ is the diagonal matrix of elemental length/area/ volume for beam/plate/solid element respectively. The $\ell_{1}$-norm $\|\boldsymbol{x}\|_{1}=\sum_{e=1}^{N}\left|x_{e}\right|$ defines the sparse regularization term and $\lambda>0$ is the corresponding regularization parameter which controls the trade-off between the sparsity and the residual norm. It has been proved that minimization of $\ell_{1}$-norm could satisfy the desired sparsity requirement[36]. Moreover, $\|\boldsymbol{x}\|_{1}$ is still convex and the linear programming techniques[36] can be easily called to solve the optimization problem in the conventional linear least squares setting. In the next section, the AM method is called to get the solution of the sparse-regularized CRE objective function (13). To get a better perspective of the sparsity-promoting nature of $\ell_{1}$-norm regularization, consider a simple structural system (see $\mathbf{F i g}$ ure 1) with displacement degree of freedoms $x$ and the potential energy of the system is designated as $f(x)$. If all masses are placed on a frictional surface with the same threshold value of the static friction force $\lambda$ (see Figure $\mathbf{1}(b)$ ), the potential energy would be changed into $f_{\lambda}(x)=f(x)+\lambda\|x\|_{1}^{1}$, which is equivalent to the $\ell_{1}$-norm regularization. As is noteworthy, if the prescribed friction $\lambda$ is greater than the maximum of the forces exerted on all masses, no movement $x=0$ would occur, while if the prescribed friction $\lambda$ is less than the $k(\ll m)$ greatest ones of the forces exerted on all masses but larger than others, $k$ masses corresponding to $k$ greatest forces would admit some movement and others keep unmoved; this leads to the sparse solution of $x$. To conclude, with a proper choice of $\lambda$, the sparsity of the solution $x$ can be reached by the $\ell_{1}-$ norm regularization and therefore, the $\ell_{1}$-norm term $\lambda\|x\|_{1}^{1}$ can indeed serve for the sparse regularization. 


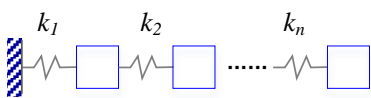

(a) No regularization

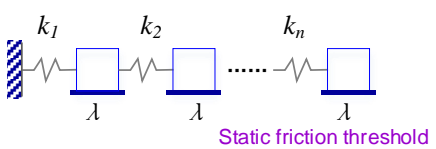

(b) Sparse regularization

Figure 1 Physical interpretation of sparse regularizations

\section{Alternating minimization method for damage identification}

\subsection{The AM method}

In this section, the AM method is proposed to practically solve this nonlinear optimization problem (13). It is shown that the AM method could lower the computational costs associated to the minimization of the CRE objective function than the traditional direct way[19]. Actually, the AM method has been a suitable approach for minimum solution of the separately convex objective function and in principle, it tries to get the solution iteratively by alternating minimization of the objective function over every separated variable in each iteration. Specifically, given the initial damage parameters $\tilde{\boldsymbol{\theta}}_{0}$ as those of the intact structure, the solution is obtained successively for $n=1,2, \ldots$ as follows

- step 1 (UMF step): Update the mechanical field $(\tilde{\boldsymbol{u}}, \tilde{\boldsymbol{q}})$ with the given damage parameters $\tilde{\boldsymbol{\theta}}^{n}$ for each given frequency

$$
\left(\tilde{\boldsymbol{u}}^{n+1}, \tilde{\boldsymbol{q}}^{n+1}\right)=\arg \min _{u \in \mathcal{U}, \boldsymbol{\sigma} \in \Im, u=\hat{u}_{k}, k \in \wp} F\left(\tilde{\boldsymbol{u}}, \tilde{\boldsymbol{q}}, \tilde{\boldsymbol{\theta}}^{n}\right)
$$

- step 2 (UDP step): Update the damage parameters $\tilde{\boldsymbol{\theta}}$ by the mechanical fields $\left(\tilde{\boldsymbol{u}}^{n+1}, \tilde{\boldsymbol{q}}^{n+1}\right)$ obtained in step 1.

$$
\tilde{\boldsymbol{\theta}}^{n+1}=\arg \min _{\tilde{\boldsymbol{\theta}}} F\left(\tilde{\boldsymbol{u}}^{n+1}, \tilde{\boldsymbol{q}}^{n+1}, \tilde{\boldsymbol{\theta}}\right)
$$

\subsection{The UMF Step}

The mechanical field $(\tilde{\boldsymbol{u}}, \tilde{\boldsymbol{q}})$ is recovered from the measured data and the given damage parameters $\tilde{\boldsymbol{\theta}}$ (given elastic matrix $\boldsymbol{D}$ ) individually for each given 
frequency. The sparse regularization term is not involved in this step. Specifically, the minimization over $\tilde{\boldsymbol{q}}$ yields the equation:

$$
\begin{aligned}
& \int_{\Omega}\left\{r_{1}\left(\boldsymbol{N}_{2}^{T} \boldsymbol{D}^{-1} \boldsymbol{N}_{2} \tilde{\boldsymbol{q}}-\boldsymbol{N}_{2}^{T} \boldsymbol{B}_{1} \tilde{\boldsymbol{u}}\right)+\right. \\
& \left.\quad r_{2}\left(\left(\rho \omega^{2}\right)^{-1} \boldsymbol{B}_{2}^{T} \boldsymbol{B}_{2} \tilde{\boldsymbol{q}}+\boldsymbol{B}_{2}^{T} \boldsymbol{N}_{1} \tilde{\boldsymbol{u}}+\left(\rho \omega^{2}\right)^{-1} \boldsymbol{B}_{2}^{T} \boldsymbol{b}\right)\right\}=0
\end{aligned}
$$

and the minimization over $\tilde{\boldsymbol{u}}$ yields

$$
\int_{\Omega}\left\{r_{1}\left(\boldsymbol{B}_{1}^{T} \boldsymbol{D} \boldsymbol{B}_{1} \tilde{\boldsymbol{u}}-\boldsymbol{B}_{1}^{T} \boldsymbol{N}_{2} \tilde{\boldsymbol{q}}\right)+r_{2}\left(\rho \omega^{2} \boldsymbol{N}_{1}^{T} \boldsymbol{N}_{1} \tilde{\boldsymbol{u}}+\boldsymbol{N}_{1}^{T} \boldsymbol{B}_{2} \tilde{\boldsymbol{q}}+\boldsymbol{N}_{1}^{T} \boldsymbol{b}\right)\right\}=0
$$

From equations (16) and (17), the mechanical field $(\tilde{\boldsymbol{u}}, \tilde{\boldsymbol{q}})$ can be updated simultaneously. Moreover, equation (17) could be simplified as

$$
\left(\begin{array}{cc}
\left(r_{1} \boldsymbol{C}_{0}+\frac{r_{2}}{\omega^{2}} \boldsymbol{H}_{0}\right) & \boldsymbol{A}_{0} \\
\boldsymbol{A}_{0}^{T} & \left(r_{1} \boldsymbol{K}_{0}+r_{2} \omega^{2} \boldsymbol{M}_{0}\right)
\end{array}\right)\left(\begin{array}{c}
\tilde{\boldsymbol{q}} \\
\tilde{\boldsymbol{u}}
\end{array}\right)=\left(\begin{array}{c}
\boldsymbol{d}_{1} \\
\boldsymbol{d}_{2}
\end{array}\right)
$$

where

$$
\begin{aligned}
\boldsymbol{C} & =\int_{\Omega} \boldsymbol{N}_{2}^{T} \boldsymbol{D}^{-1} \boldsymbol{N}_{2} d \Omega \\
\boldsymbol{H} & =\int_{\Omega} \frac{1}{\rho} \boldsymbol{B}_{2}^{T} \boldsymbol{B}_{2} d \Omega, \\
\boldsymbol{M} & =\int_{\Omega} \rho \boldsymbol{N}_{1}^{T} \boldsymbol{N}_{1} d \Omega \\
\boldsymbol{K} & =\int_{\Omega} \boldsymbol{B}_{1}^{T} \boldsymbol{D} \boldsymbol{B}_{1} d \Omega \\
\boldsymbol{A} & =\int_{\Omega}\left(-r_{1} \boldsymbol{N}_{2}^{T} \boldsymbol{B}_{1}+r_{2} \boldsymbol{B}_{2}^{T} \boldsymbol{N}_{1}\right) d \Omega \\
\boldsymbol{d}_{1} & =\int_{\Omega}\left\{-r_{2}\left(\rho \omega^{2}\right)^{-1} \boldsymbol{B}_{2}^{T} \boldsymbol{b}-\left(r_{1} \boldsymbol{N}_{2}^{T} \boldsymbol{D}^{-1} \boldsymbol{N}_{2}+r_{2}\left(\rho \omega^{2}\right)^{-1} \boldsymbol{B}_{2}^{T} \boldsymbol{B}_{2}\right) \tilde{\boldsymbol{q}}_{p}\right\} d \Omega, \\
\boldsymbol{d}_{2} & =\int_{\Omega}\left\{-r_{2} \boldsymbol{N}_{1}^{T} \boldsymbol{b}-\left(-r_{1} \boldsymbol{B}_{1}^{T} \boldsymbol{N}_{2}+r_{2} \boldsymbol{N}_{1}^{T} \boldsymbol{B}_{2}\right) \tilde{\boldsymbol{q}}_{p}\right\} d \Omega .
\end{aligned}
$$

In equation (19), $\boldsymbol{A}_{0}, \boldsymbol{C}_{0}, \boldsymbol{K}_{0}, \boldsymbol{M}_{0}$ and $\boldsymbol{H}_{0}$ are the reduced forms of $\boldsymbol{A}, \boldsymbol{C}, \boldsymbol{K}$,

$\boldsymbol{M}$ and $\boldsymbol{H}$ after applying the homogeneous boundary conditions.

To enforce the incomplete measured data, the displacements vector $\tilde{\boldsymbol{u}}$ will now be partitioned symbolically into unmeasured displacement $\tilde{\boldsymbol{u}}^{U}$ and measured displacement $\hat{\boldsymbol{u}}^{M}$. For the sake of convenience, in the following equations 
superscript $U$ and $M$ represent the corresponding unmeasured and measured DOFs of the array respectively. As a result, equation (18) can be rewritten as

$$
\boldsymbol{L}(\tilde{\boldsymbol{\theta}})\left(\tilde{\boldsymbol{q}}, \tilde{\boldsymbol{u}}^{U}\right)^{T}=\boldsymbol{S}(\tilde{\boldsymbol{\theta}})
$$

where

$$
\begin{aligned}
& \boldsymbol{L}(\tilde{\boldsymbol{\theta}})=\left(\begin{array}{cc}
r_{1} \boldsymbol{C}_{0}+\frac{r_{2}}{\omega^{2}} \boldsymbol{H}_{0} & \boldsymbol{A}_{0} \\
\boldsymbol{A}_{0}^{T^{U}} & \left(r_{1} \boldsymbol{K}_{0}+r_{2} \omega^{2} \boldsymbol{M}_{0}\right)^{U U}
\end{array}\right) \\
& \boldsymbol{S}(\tilde{\boldsymbol{\theta}})=\left(\boldsymbol{d}_{1},\left(\boldsymbol{d}_{2}^{\prime}\right)_{n}\right)^{T}, \\
& \boldsymbol{d}_{2}^{\prime}=\boldsymbol{d}_{2}^{U}-\left(r_{1} \boldsymbol{K}_{0}+r_{2} \omega^{2} \boldsymbol{M}_{0}\right)^{U M} \hat{\boldsymbol{u}}^{M}
\end{aligned}
$$

\subsection{The UDP Step}

Due to the decoupling of the CRE objective function (7) as mentioned in Section2.4, the UDP step is tackled by solving a series of single-variable optimization problems, that is, minimization of equation (13) with respect to $\theta_{e}$ for each element $e(e=1,2, \ldots, N)$ takes the form

$$
\begin{aligned}
& \int_{\Omega^{e}} \frac{r_{1}}{2}\left\{\left(\boldsymbol{N}_{2} \tilde{\boldsymbol{q}}^{e}\right)^{T} \frac{\left(\boldsymbol{D}_{e}\right)^{-1}}{\left(\theta_{e}\right)^{2}}\left(\boldsymbol{N}_{2} \tilde{\boldsymbol{q}}^{e}\right)-\left(\boldsymbol{B}_{1} \tilde{\boldsymbol{u}}^{e}\right)^{T} \boldsymbol{D}_{e}\left(\boldsymbol{B}_{1} \tilde{\boldsymbol{u}}^{e}\right)\right\} d x \\
& -\lambda \Delta^{e}\left\{\operatorname{sign}\left(\theta_{e}-1\right)\right\} d x=0 .
\end{aligned}
$$

Finally, one has

$$
\theta_{e}=\left\{\begin{array}{l}
\sqrt{\frac{b_{e}}{a_{e}+\lambda \Delta^{e}}}, \text { if } \lambda<\Lambda_{e} \\
\sqrt{\frac{b_{e}}{a_{e}-\lambda \Delta^{e}}}, \text { if } \lambda<-\Lambda_{e} \quad, e=1,2, \ldots, N \\
1, \text { if } \lambda \geq\left|\Lambda_{e}\right|
\end{array}\right.
$$

where

$$
\begin{aligned}
\Lambda_{e} & =\frac{1}{\Delta^{e}} \int_{\Omega_{e}} \frac{r_{1}}{2}\left\{\left(\boldsymbol{N}_{2} \tilde{\boldsymbol{q}}^{e}\right)^{T}\left(\boldsymbol{D}_{e}\right)^{-1}\left(\boldsymbol{N}_{2} \tilde{\boldsymbol{q}}^{e}\right)-\left(\boldsymbol{B}_{1} \tilde{\boldsymbol{u}}^{e}\right)^{T} \boldsymbol{D}_{e}\left(\boldsymbol{B}_{1} \tilde{\boldsymbol{u}}^{e}\right)\right\} d \Omega \\
& =\frac{1}{\Delta^{e}}\left(b_{e}-a_{e}\right)
\end{aligned}
$$

and

$$
a_{e}=\int_{\Omega_{e}} \frac{r_{1}}{2}\left(\boldsymbol{B}_{1} \tilde{\boldsymbol{u}}^{e}\right)^{T} \boldsymbol{D}_{e}\left(\boldsymbol{B}_{1} \tilde{\boldsymbol{u}}^{e}\right) d \Omega, b_{e}=\int_{\Omega_{e}} \frac{r_{1}}{2}\left(\boldsymbol{N}_{2} \tilde{\boldsymbol{q}}^{e}\right)^{T}\left(\boldsymbol{D}_{e}\right)^{-1}\left(\boldsymbol{N}_{2} \tilde{\boldsymbol{q}}^{e}\right) d \Omega
$$


Datailed explanations of $\Lambda_{e}$ are discussed in the next section. Based on equation (23), the value of $\theta_{e}$ is found to be prone to be unchanged from the initial value 1 when $\left|\Lambda_{e}\right| \leq \lambda$ while $\theta_{e}$ begin to deviate from 1 to $\hat{\theta}_{e}$ when $\left|\Lambda_{e}\right| \geq \lambda$. That is to say, the regularization parameter $\lambda$ determines whether damage occurs: as long as $\lambda<\left|\Lambda_{e}\right|$, the $e$ th element would admit stiffness change or damage; otherwise if $\lambda \geq\left|\Lambda_{e}\right|$, the damage would be found to not occur in the eth element.

Moreover, from equations (22) and (23), the sparse regularization term would introduce little computational complexity and the minimization over each damage parameter along with the sparse regularization term is solved immediately by the closed-form solution in equation (23). This advantage is benefit from the decoupling of the CRE objective function with respect to the damage parameters.

\subsection{Regularization Parameter Estimation}

As shown in many references $[37,38]$, the regularization parameter $\lambda$ plays an important role in the quality of the damage identification results, especially in cases where high levels of noise are present in the data. In this section, a novel and simple strategy, named 'threshold setting method', for estimating the optimal regularization parameter $\lambda_{\text {est }}$, is devised.

For each iterative step, $\boldsymbol{\Lambda}=\left\{\left|\Lambda_{e}\right|, e=1,2, \ldots, N\right\}$ could be obtained for each element by equation (24). In fact, $\lambda$ is used to distinguish the damaged elements from the undamaged elements and $\left|\Lambda_{e}\right|$ reflects the difference between the stiffness parameter obtained directly from the CRE objective function without sparsity $\left(\hat{\theta}_{e}\right)$ and the undamaged stiffness parameter $\left(\theta_{e}=1\right)$ for each element. It is easily known that if this difference is large enough $\left(\left|\Lambda_{e}\right|>\lambda\right)$, damage is reasonably assumed to occur. In other words, elements with higher values of $\left|\Lambda_{e}\right|$ are likely to be damaged with stronger possibility while lower values indicate undamage and perturbation errors. Thus, setting one of the values in $\boldsymbol{\Lambda}$ as threshold could separate the damaged and undamaged elements.

As a result, a simple procedure called 'the threshold setting method' to estimate the optimal regularization parameter $\lambda_{\text {est }}=\operatorname{TSM}\left(\boldsymbol{\Lambda}, l_{\max }, \alpha\right)$ is proposed 
for each iterative step as follows:

1. Get $\boldsymbol{\Lambda}=\left\{\left|\Lambda_{e}\right|, e=1,2, \ldots, N\right\}$ from equation (24) and sort the absolute values in the descending order, leading to $\left\{\hat{\Lambda}_{1} \geq \hat{\Lambda}_{2} \geq \ldots\right\}$;

2. Fix the maximum threshold setting rank $l_{\max }$ and the discriminating ratio $\alpha$

3. Obtain the optimal regularization parameter $\lambda_{\text {est }}$ : for each $l=1, \ldots, l_{\max }-$ 1 ,

$$
\lambda_{\text {est }}= \begin{cases}\hat{\Lambda}_{l+1}, & \text { if } \hat{\Lambda}_{l} \geq \alpha \cdot \hat{\Lambda}_{l+1} \\ \hat{\Lambda}_{l_{\max }}, & \text { else }\end{cases}
$$

The maximum threshold setting rank $l_{\max }$ limits the maximum number of possible damages and principally one tends to choose a relatively small value of $l_{\max }$ to guarantee the sparsity of the identification results. On the other hand, it is conceivable that the general undamaged elements have relatively small values of $\left|\Lambda_{e}\right|$ corresponding to the noise level and would be quite away from $\left|\Lambda_{e}\right|$ of the damaged elements. As a result, the discrimination ratio $\alpha$ measures the distance between the minimum error caused by possible damages and the maxi-

than $\hat{\Lambda}_{l+1}\left(\hat{\Lambda}_{l} \geq \alpha \cdot \hat{\Lambda}_{l+1}\right)$, element $l$ is identified as damaged. The maximum threshold setting rank $l_{\max }$ gives possible maximum number (initial guess) of damages while the discrimination ratio $\alpha$ narrow it down. More discussions on the choice of $\left(l_{\max }, \alpha\right)$ could be found in Section 4.1.

\subsection{Summary of the AM method}

By combining the UMF step in Section 3.2 and the UDP step in Section 3.3 , using the proposed threshold setting method so as to estimate the optimal regularization parameter $\lambda$ in Section 3.4, the damage identification algorithm by min-CRE and sparse regularization can be established as shown in Table 1. It should be noted that different analysis models could be applied in the UMF step and the UDP step. In the UMF step, more elements are recommended so 
as to avoid the discretization error for the mechanical field analysis, while less elements could be used in the UDP step to mitigate the ill-posed problem if acquiring less damage parameters. Nevertheless, as the sparse regularization could indeed circumvent the possible ill-posedness caused by more damage parameters, refined elements for the UDP step is also possible in the proposed damage identification approach, which would be illustrated in the following numerical examples.

\section{Numerical simulation}

In the following, three numerical simulations are used to test the proposed sparse-regularized min-CRE approach using FRF data. Damage identification results obtained by three identification approaches are compared in Section 4.1 and Section 4.2, namely the min-CRE approach with sparse regularization $(\mathrm{C} 1)$, the sensitivity approach with Tikhonov regularization (S2) and the sensitivity approach with sparse regularization (S1). The advantage of being efficient for the proposed min-CRE approach is strongly substantiated. The example in Section 4.1 further considers some modeling errors in the mass matrix so as to testify the robustness of the identification results. Section 4.3 shows that the sparse regularization can significantly improve the accuracy of the identification results for a more general three-dimensional structure. For application of the iteration algorithm, the convergence tolerance is practically set to $\mathrm{TOL}=1 \times$ $10^{-6}$.

\subsection{A simply supported beam structure}

In this example, a simply supported beam is utilized to compare the accuracy and efficiency between the proposed min-CRE approach and sensitivity approaches with different regularization techniques. The total length of the beam is $1 \mathrm{~m}$ and the mass-per-length and bending stiffness are estimated as $1 \mathrm{~kg} / \mathrm{m}$ and $1 \mathrm{~N} \cdot \mathrm{m}^{2}$, respectively. As with finite element modelling, the beam is uniformaly divided into 16 Eular-Bernoulli beam elements with 17 nodes each 
Table 1 Summery of the proposed AM method

Initial algorithm setting

$\circ$ set inital damage parameters $\tilde{\boldsymbol{\theta}}_{(0)}=\tilde{\boldsymbol{\theta}}_{0}$ as those of the intact structure

$\circ$ set values of the maximum threshold setting rank $l_{\max }$ and the discriminating ratio $\alpha$ for the threshold setting method

$\circ$ define the convergence tolerance TOL and the maximum number of iterations $Z_{\max }$

- load the measured FRF data

Alternating minimization approach (for $j=1: Z_{\max }$ )

- Refer to the UMF step in Section 3.2 to obtain the updating mechanical field $\left(\tilde{\boldsymbol{q}}, \tilde{\boldsymbol{u}}^{U}\right)_{(j)}^{T}=\boldsymbol{L}\left(\tilde{\boldsymbol{\theta}}_{(j-1)}\right)^{-1} \boldsymbol{S}\left(\tilde{\boldsymbol{\theta}}_{(j-1)}\right)$

○ Choose the optimal regularization parameter $\lambda_{\text {est }}$ according to the treshold setting method in Section 3.4 from

- for $e=1: N$

- $a_{e}=\int_{\Omega_{e}} \frac{r_{1}}{2}\left(\boldsymbol{B}_{1} \tilde{\boldsymbol{u}}_{(j)}^{e}\right)^{T} \boldsymbol{D}_{e}\left(\boldsymbol{B}_{1} \tilde{\boldsymbol{u}}_{(j)}^{e}\right) d \Omega$,

$-b_{e}=\int_{\Omega_{e}} \frac{r_{1}}{2}\left(\boldsymbol{N}_{2} \tilde{\boldsymbol{q}}_{(j)}^{e}\right)^{T}\left(\boldsymbol{D}_{e}\right)^{-1}\left(\boldsymbol{N}_{2} \tilde{\boldsymbol{q}}_{(j)}^{e}\right) d \Omega$

$-\Lambda_{e}=\frac{1}{\Delta^{e}}\left(b_{e}-a_{e}\right)$, end for

$-\lambda_{\text {est }}=\operatorname{TSM}\left(\boldsymbol{\Lambda}, l_{\max }, \alpha\right)$

- Refer to the UDP step in Section 3.3 to obtain the updating damage parameter $\theta_{e(j)}$ for each element:

- if $\lambda_{\text {est }}<\Lambda_{e}$ then $\theta_{e(j)}=\sqrt{\frac{b_{e}}{a_{e}+\lambda_{\text {est }} \Delta^{e}}}$,

- else if $\lambda_{\text {est }}<-\Lambda_{e}$ then $\theta_{e(j)}=\sqrt{\frac{b_{e}}{a_{e}-\lambda_{\text {est }} \Delta^{e}}}$,

- else $\theta_{e(j)}=\theta_{e 0}=1$

- Convergence criterion

- if $\left\|\tilde{\boldsymbol{\theta}}_{(j)}-\tilde{\boldsymbol{\theta}}_{(j-1)}\right\| /\left\|\tilde{\boldsymbol{\theta}}_{(0)}\right\| \leq$ TOL break. 

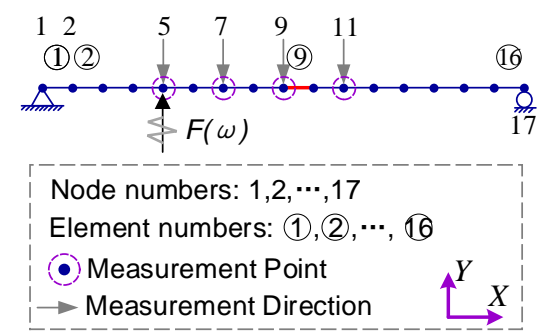

Figure 2 Model of simply supported beam structure

with two degrees of freedom (see Figure $\mathbf{2}$ for more details). The damage scenario admits the damage in element 9 with $20 \%$ stiffness reduction. The measurement points are chosen to be node 5, 7, 9 and 11 (Y direction only). The selected frequency range is simply chosen to be $[1,80] \mathrm{Hz}$ with $1 \mathrm{~Hz}$ intervals. For a more complicated model, an appropriate choice of the selected frequency range could improve the identification results greatly. Detailed selecting rules are discussed in Section 4.2. The measured FRF data at $k$ th selected excitation frequency is obtained from simulation with addition of the measurement noise as follows,

$$
\operatorname{noise}_{k}(s)=\operatorname{randn} \cdot a \cdot d_{k}(s), k=1,2, \ldots m
$$

where randn is the normal distribution with zero mean and unit deviation,

$a$ is the applied noise level and $d_{k}(s)$ is the measurement FRF data at certain position $s$. Noise level of $10 \%$ (Signal-to-Noise-Ratio, $\mathrm{SNR}=10 \mathrm{~dB}$ ) is considered in this example. For excitation, an unit harmonic load as shown in Figure 2 is enforced at node 5 .

\subsubsection{On choice of $\left(l_{\max }, \alpha\right)$ in the threshold setting method}

the discriminating ratio $\alpha$ work in the threshold setting method, different choices of $\left(l_{\max }, \alpha\right)$ for damage identification in different noise levels are studied herein and detailed results are displayed in Figure 3. On one hand, the identification process tends to be reasonably robust for a broad selection of $\left(l_{\max }, \alpha\right)$ with appropriate ranges of $\alpha \in[2,50]$ and $l_{\max } \in[2,10]$ for the case with low level 


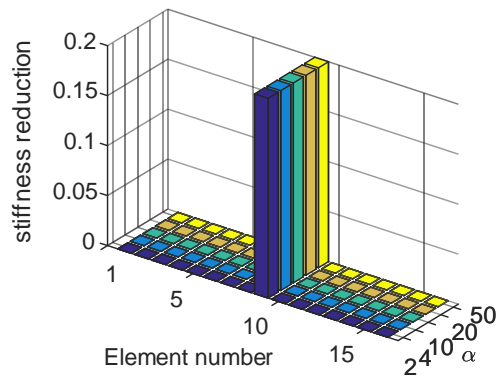

(a) Fixing $I_{\max }=5$ and varying $\alpha$ (no noise)

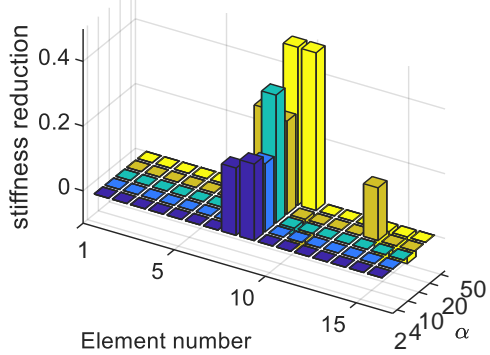

(c) Fixing $I_{\max }=5$ and varying $\alpha(10 \%$ noise $)$

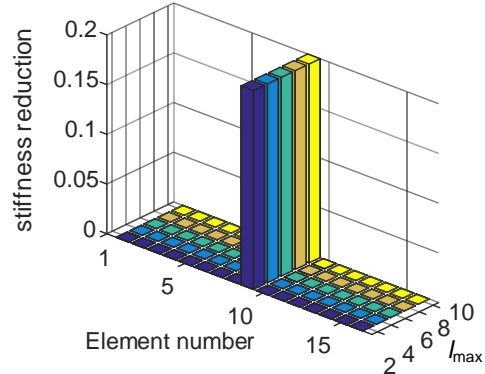

(b) Fixing $\alpha=4$ and varying $I_{\max }$ (no noise)

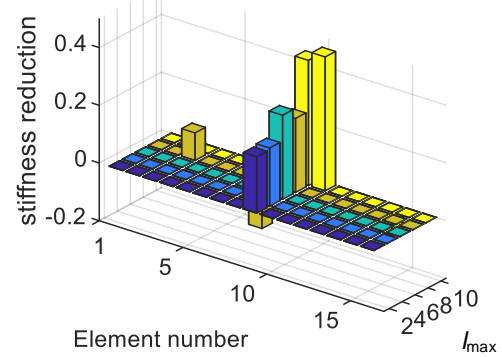

(d) Fixing $\alpha=4$ and varying $I_{\max }(10 \%$ noise $)$

Figure 3 Damage identification results under different choice of $\left(l_{\max }, \alpha\right)$

of noise (Figure 3(a), (b)). On the other hand, the choice of $\left(l_{\max }, \alpha\right)$ should be paid more attention for the case with high level of noise (Figure 3(c), (d)). Damage locations are badly identified for $\alpha=2$ and $\alpha \geq 20$. This means that the discriminating ratio $\alpha$ should not be too small or too large. In terms

of the maximum threshold setting rank $l_{\max }, l_{\max } \geq 8$ leads to the failure of damage identification, indicating that $l_{\max }$ shall not be too large. As a result, in the following of this example, the threshold parameters are reasonably fixed at $l_{\max }=5, \alpha=4$.

\subsubsection{Comparison to the results by sensitivity-based algorithms}

In this section, the following sensitivity-based algorithm, representing probably the most widely used techniques on the use of FRF data is considered.

Normally, the damage identification process based on the sensitivity approach is formulated as minimization of the following objective function-weighted 
least squares of the error in measured quantities,

$$
\arg \min \|\hat{\boldsymbol{R}}-\boldsymbol{R}(\boldsymbol{\theta})\|_{W}^{2}
$$

where $\hat{\boldsymbol{R}}$ and $\boldsymbol{R}(\boldsymbol{\theta})$ denotes the measured and analytically predicted outputs respectively. $\boldsymbol{W}$ is the positive definite weight matrix. Linearization of the above non-linear problem (28) at given $\overline{\boldsymbol{\theta}}$ for iterative method is as follows

$$
\hat{\boldsymbol{R}}-\boldsymbol{R}(\boldsymbol{\theta})=\Delta \boldsymbol{R}(\overline{\boldsymbol{\theta}})-\boldsymbol{S}(\overline{\boldsymbol{\theta}}) \Delta \boldsymbol{\theta}
$$

where $\Delta \boldsymbol{\theta}:=\boldsymbol{\theta}-\overline{\boldsymbol{\theta}}$ is the update of $\boldsymbol{\theta}, \Delta \boldsymbol{R}(\overline{\boldsymbol{\theta}}):=\hat{\boldsymbol{R}}-\boldsymbol{R}(\overline{\boldsymbol{\theta}})$ is the residual and $\boldsymbol{S}(\overline{\boldsymbol{\theta}})$ is the response sensitivity matrix. For the method using frequency response function, the mathematical formulation of $\boldsymbol{R}(\boldsymbol{\theta})$ and the eth column of sensitivity matrix $\boldsymbol{S}$ are often expressed as[39]:

$$
\begin{gathered}
\boldsymbol{R}(\boldsymbol{\theta})=\left[-\omega^{2} \boldsymbol{M}(\boldsymbol{\theta})+\boldsymbol{K}(\boldsymbol{\theta})\right] \hat{\boldsymbol{u}}^{M} \\
\boldsymbol{S}_{e}=\left.\frac{\partial \boldsymbol{R}(\boldsymbol{\theta})}{\partial \theta_{e}}\right|_{\theta=\bar{\theta}}=\left[-\omega^{2} \frac{\partial \boldsymbol{M}}{\partial \theta_{e}}+\frac{\partial \boldsymbol{K}}{\partial \theta_{e}}\right]_{\theta=\bar{\theta}} \hat{\boldsymbol{u}}^{M}
\end{gathered}
$$

where $\hat{\boldsymbol{u}}^{M}$ represents the measured frequency-domain displacement response. Static condensation is needed so as to condense the above equation to the measured degrees of freedom and the weighting matrix $\boldsymbol{H}(\omega, \bar{\theta})$, the frequency response function matrix should also be considered so as to reduce the bias on the estimated parameters. To circumvent the ill-posed problem, regularization is required. Objective function, solution method for each iteration and regularization parameter estimation method for different regularization types are briefly summarized in Table 2.

The final identification results are presented in Figure 4. As is seen, all of these three approaches can well identify the damage location and extent in the beam through of $10 \%$ noise level, except that false positions, although with minor degree, are also observed by the sensitivity approach with Tikhonov regularization (S2). Many researches have shown that Tikhonov regularization tends to produce over-smooth solutions because the quadratic regularizer cannot recover the sparse features of the solution. 
As regards the corresponding convergence property in Table 3, it is shown that the computational effort necessary to solve $\mathrm{C} 1(0.51 \mathrm{~s})$ is much less than the sensitivity approaches(4.89s-899.47s), although it takes more steps for $\mathrm{C} 1$ to get

convergent. This advantage attributes to the inherent decoupling nature of the CRE function with respect to the damage parameters, making every parameterupdate-step solved as a series of single-variable optimization problems. It should be noted that due to the lack of closed-form solution for regularization parameter estimation, the computational time for S1 is huge(899.47s). For S1 with unknown $\lambda$, an appropriate range of $\lambda(2.9-5.4)$ is picked up by achieving balance between the residual norm and solution norm[29] as shown in Figure 5(b). With the properly chosen or experienced-based $\lambda$, the computational cost of S1 could be reduced significantly $(4.89 \mathrm{~s})$.

\subsubsection{Considering modeling errors}

With respect to the modeling errors, simple mass modeling errors are added to the simulated beam structure. To do so, the exact mass density of element 2, 3 and 12, which are involved into the generation of the measured FRF data through simulation, are changed to $1.05 \mathrm{~kg} / \mathrm{m}, 1.05 \mathrm{~kg} / \mathrm{m}$ and $0.95 \mathrm{~kg} / \mathrm{m}$ respectively, while the mass density for damage identification are still set to be $1 \mathrm{~kg} / \mathrm{m}$ as before. Noise level of $10 \%$ is also added into the measured FRF data. The identification results obtained by the three approaches are shown in Figure 6. As could be seen, the present approach (C1) could still identify the damage location clearly, although with a less satisfatory damage extent assessment. However, this bias can be further revised if needed by simply fixing the damage location and optimizing the extent to match the observed changes. For the sensitivity-based approaches (S2\&S1), the modelling errors may deteriorate the damage identification if no additional improvement, such as optimizing the weight matrix through the model error covariance[40], is enforced. 
Table 2 Sensitivity-based identification approaches with different regularizations

\begin{tabular}{cccc}
\hline Approach & $\begin{array}{c}\text { Objective function } \\
\text { for each iteration }\end{array}$ & $\begin{array}{c}\text { Solution method } \\
\text { for each iteration }\end{array}$ & $\begin{array}{c}\text { Parameter estimation } \\
\text { method }\end{array}$ \\
\hline \multirow{2}{*}{ S2[39] } & $\|\Delta \boldsymbol{R}(\overline{\boldsymbol{\theta}})-\boldsymbol{S}(\overline{\boldsymbol{\theta}}) \Delta \boldsymbol{\theta}\|_{\boldsymbol{W}}^{2}+$ & closed-form solution & L-curve method \\
& $\lambda\|\Delta \boldsymbol{\theta}\|_{2}^{2}$ & $\Delta \boldsymbol{\theta}=\left[\boldsymbol{S}(\overline{\boldsymbol{\theta}})^{T} \boldsymbol{W} \boldsymbol{S}(\overline{\boldsymbol{\theta}})+\lambda \boldsymbol{I}\right]^{-1} \boldsymbol{S}(\overline{\boldsymbol{\theta}})^{T} \boldsymbol{W} \Delta \boldsymbol{R}(\overline{\boldsymbol{\theta}})$ & \\
\hline \multirow{2}{*}{ S1[22] } & $\|\Delta \boldsymbol{R}(\overline{\boldsymbol{\theta}})-\boldsymbol{S}(\overline{\boldsymbol{\theta}}) \Delta \boldsymbol{\theta}\|_{\boldsymbol{W}}^{2}+$ & Primal-dual interior point method & Using residual and \\
& $\lambda\|\Delta \boldsymbol{\theta}\|_{1}$ & Newton's method (again iterative) proceeds & solution norms[29] \\
\hline
\end{tabular}

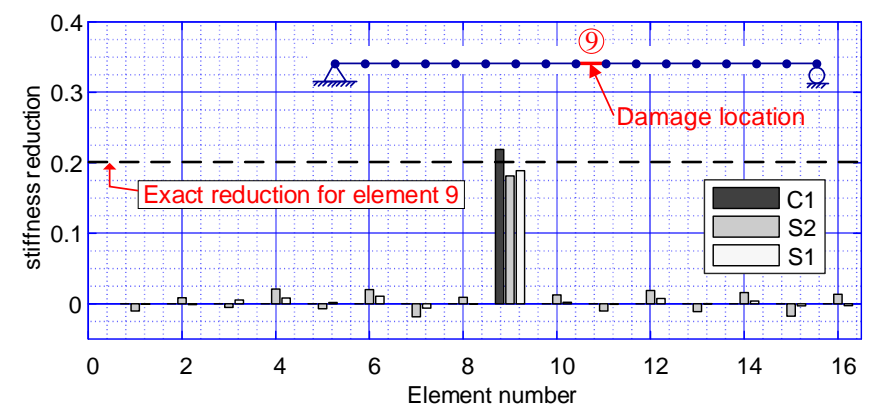

Figure 4 Stiffness reduction for each element using different approaches

Table 3 Convergence property for different identification approaches

\begin{tabular}{ccc}
\hline Approach & Numbers of iterations & Total computation time (s) \\
\hline C1 & 24 & 0.51 \\
S2 & 6 & 5.41 \\
S1 & - & 899.47 \\
S1 (with known $\lambda)$ & 9 & 4.89 \\
\hline
\end{tabular}



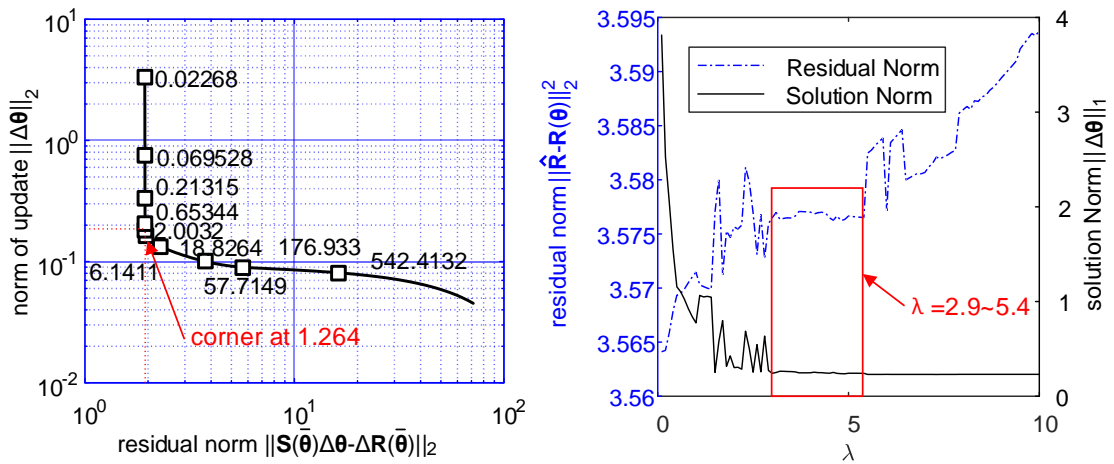

Figure 5 Regularization parameter selection: (a) L-curve method for Tikhonov regularization; (b) using residual and solution norms for sparse regularization

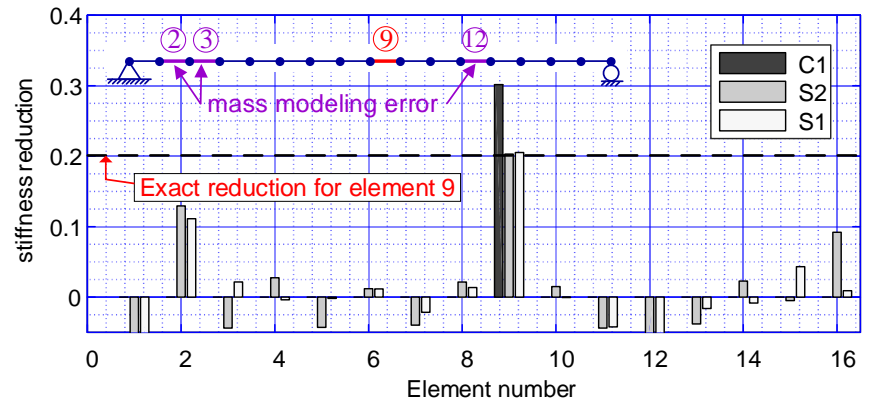

Figure 6 Stiffness reduction for each element using different approaches with mass modeling errors 


\subsection{A plane frame structure}

A plane frame structure as shown in Figure 7 is studied in the second example (see reference[9] for detailed explanation on the target structure). The height and width of the frame are $H=1.2 \mathrm{~m}$ and $L=0.6 \mathrm{~m}$, respectively, and the rectangular cross-sectional dimensions are $b=0.01 \mathrm{~m}$ and $h=0.02 \mathrm{~m}$ with the inertial moment for planar bending $I=6.67 \times 10^{-9} \mathrm{~m}^{4}$ and area $A=2 \times 10^{-4} \mathrm{~m}^{2}$. For the undamaged frame, the mass density of the material is $\rho=2.7 \times 10^{3} \mathrm{~kg} / \mathrm{m}^{3}$ and the Young's modulus is $E=6.9 \times 10^{10} \mathrm{~N} / \mathrm{m}^{2}$. The plane frame analysis model consists of 88 Euler-Bernoulli beam elements with 89 nodes, each with three degrees of freedom. This finer numerical model is used to obtain the mechanical field for the UMF step so that the discretization error caused by finite element model could be ignored. For the UMP step, the structure is divided in 11 sets of elements (see Figure $7(\mathrm{~b})$ ) and the identification procedure updates the 11 element parameters independently. A single harmonic load is applied in the $x$-direction of node 2 . The FRF data are obtained by the discrete Fourier transform(DFT) of the corresponding numerical simulated accelerations (see Figure 9 for the FRF data of node 2). Twelve damage scenarios, including single and multiple, small and large damage, unpolluted and polluted measurements are taken into account, as listed in Table 4, where Nil stands for no noise. The measurement locations and the corresponding directions are also illustrated in Table 4 and Figure 8. Herein, the stiffness parameters $\left\{\theta_{e}\right.$, $e=1,2, \ldots, N\}$, standing for the Young's modulus of each element set, is identified and the identified results in the percentage error forms are presented as

$$
\text { Relative error }(\%)=\frac{\theta_{e}^{i d}-\theta_{e}}{\theta_{e}} \times 100 \%
$$

350 each element set, respectively. Noise level of $10 \%(\mathrm{SNR}=10 \mathrm{~dB})$ is added to the measurements primarily.

Several practical rules for selecting excitation frequencies are

- Excitation frequencies should not be selected at resonance frequencies to 
prevent resonance phenomena and high sensitivity to noise.

- Dynamic response of the target structure is more sensitive to damages for frequency points close to resonances than points far from resonances. Therefore, excitation frequency points near resonance frequencies are recommended for measurement[32].

- Although the response of the target structure in higher frequency ranges, which excites higher mode shapes, is more sensitive to damages, numerical analysis results are shown with less accuracy than those in lower frequency ranges[41].

- Using more frequency ranges corresponding to different resonance frequencies could improve the identification accuracy while using more frequency points near one resonance frequency seems to have no significantly effect on the results for lower noise level cases.

- For higher levels of noise, presence of more frequency points near certain resonance frequency can result in a robust identification.

Based on these rules, the selected frequency ranges are given in Table $\mathbf{5}$ and the sampling frequency stepping size in each frequency range is $4 \mathrm{~Hz}$. Consequently, identification results of each scenario are obtained using 50 frequency points.

As is observed in Table 6 with the relative error defined in equation (32), for the single damage scenarios 1-6, the proposed approach could provide satisfied identification results: the maximum relative error does not exceed $0.02 \%$ in the case of no noise and $1 \%$ in the case of $10 \%$ noise. Only the horizontal acceleration response at nodes 2 and 9 is measured herein. For the multiple damages scenarios 7-12, the horizontal frequency response at node 4 and the vertical acceleration response at node 7 are additionally measured. The identification by the min-CRE approach can well identify the damage locations as well as extents with a maximum relative error $0.01 \%$ in the case of no noise and $3.7 \%$ in the case of $10 \%$ noise. Compared with the results in the reference[9], which 
used an enhanced response sensitivity approach with the Tikhonov regularization under the same measurement degrees of freedom, identification results of the min-CRE approach with the sparse regularization demonstrated the superiority for all of the 12 scenarios. Most of this superior identification accuracy is attributed to the enforced sparse regularization: the sparse regularization yields sparse damages that are more compatible with pratical damage patterns, while the Tikhonov-regularized damage identification does not result in sparse solutions, which typically have non-zero relative errors associated with all damage parameters[42]. All coincide with the existed observations where replacement of the Tikhonov regularization by the sparse regularization can indeed improve the identification accuracy and robustness for damage identification.

To get a better picture on the effectiveness of the sparse regularization, detailed identification results for scenario 5 are shown in Figure 10. It is shown that stiffness reduction is obtained based on $\hat{\theta}_{e}$ in equation (??), where no sparsity term is introduced. Obviously, when there is no measurement noise, using or not using sparse regularization would lead to the same good identification results. However, it is almost impossible to get to the reasonable identification results for the case without sparse regularization when measurement noise is enforced. It is clear that the sparse regularization greatly improves the identifiability and robustness of the CRE-approach. Furthermore, to visualize how the sparse-regularized min-CRE approach proceeds, the iterative procedure for the scenario 5 is shown in Figure 11. On the other hand, Figure 12 compares the evolution of the constitutive relation error(CRE) for each of the iterative step with different levels of noise $(0 \%, 5 \%, 10 \%$ and $20 \%)$ for damage scenario 5. As is observed, the error decreases with the number of iterations increases, verifying the convergence of the present method. 

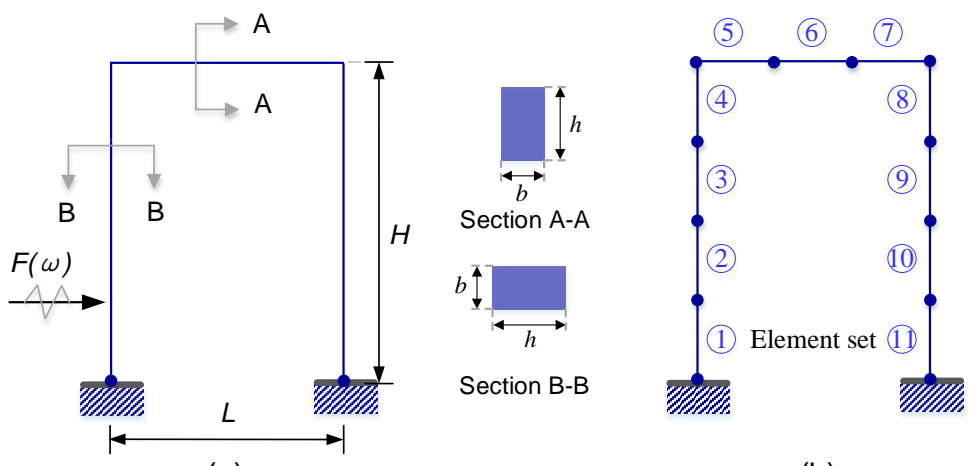

(a)

(b)

Figure 7 Model of plane frame structure: (a)Geometry, (b)Element sets

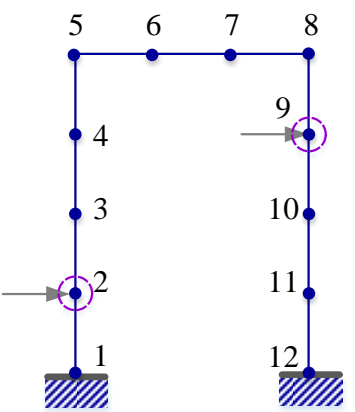

(a)

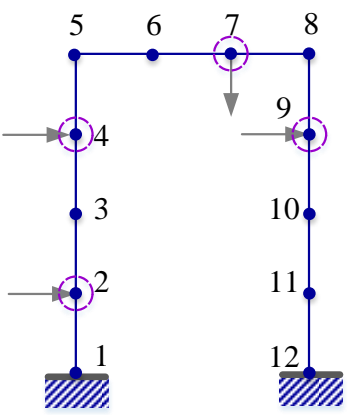

(b)

$\lceil$ (e) Measurement Point $\rightarrow$ Measurement Direction $\mid$

Figure 8 Measurement points and directions 


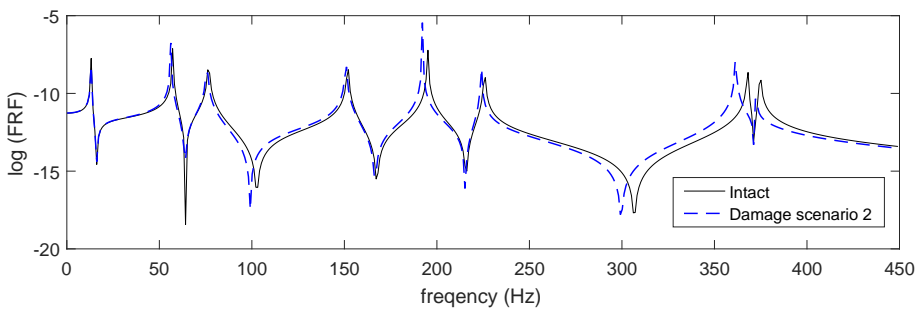

(a) Scenario 2

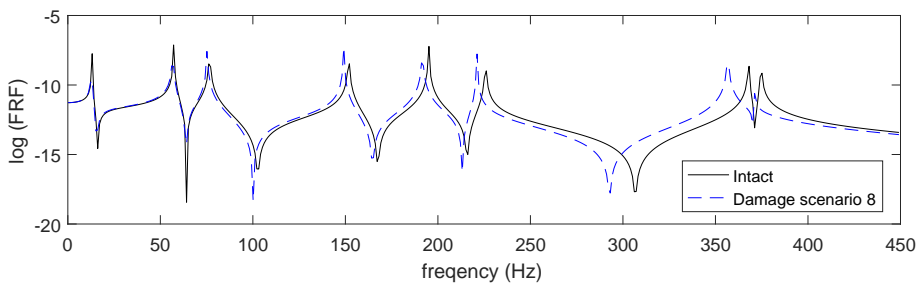

(b) Scenario 8

Figure $9 \mathrm{FRF}$ of the plane frame structure in the $x$-direction of node 2
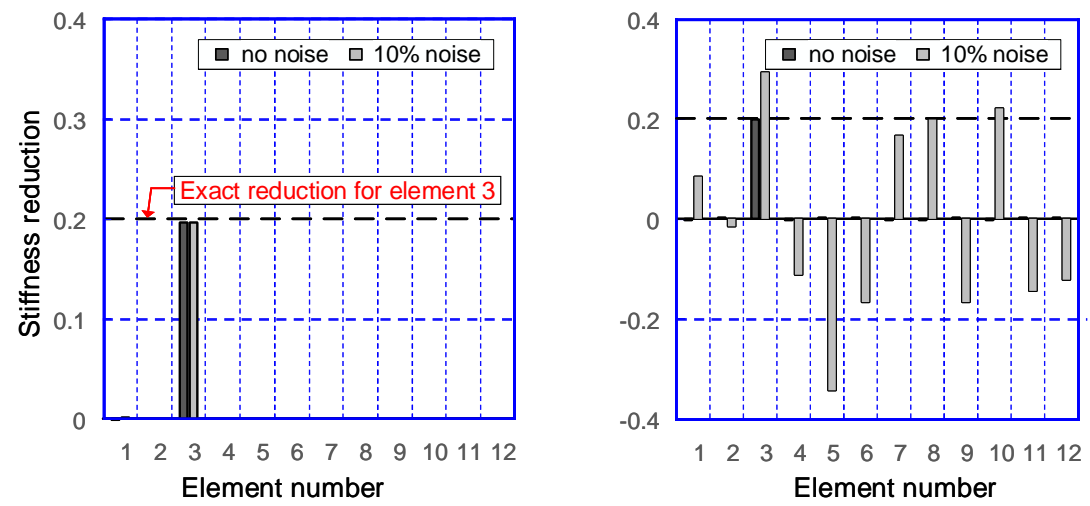

Figure 10 Stiffness reduction for scenario 2/5: (a) the min-CRE approach with sparse regularization; (b) the min-CRE approach without sparse regularization 


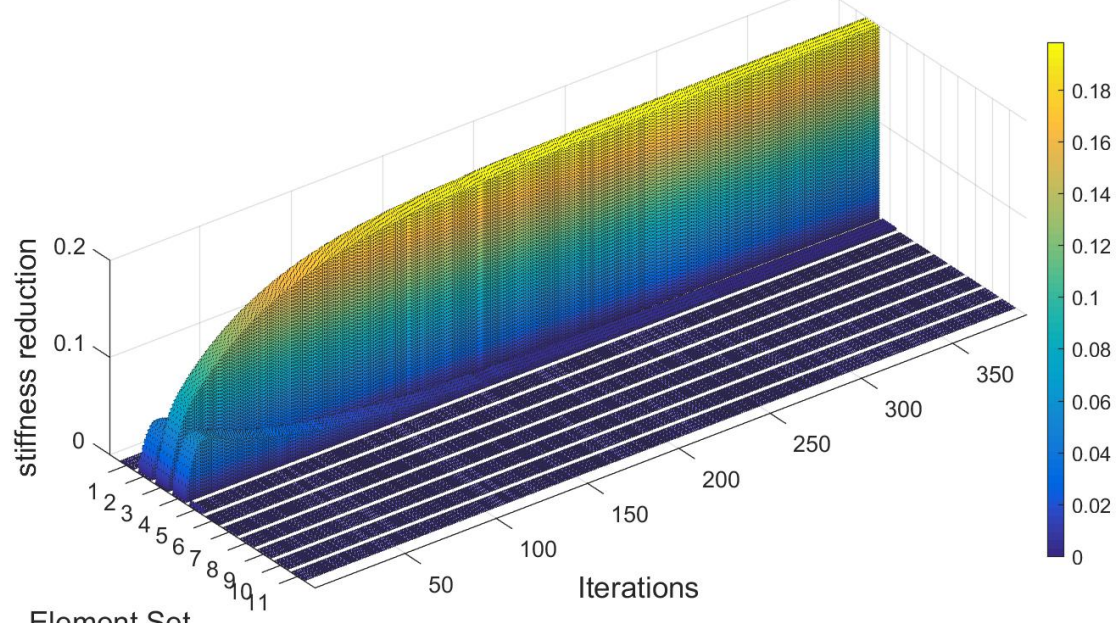

Figure 11 Damage identification procedure for scenario 5 of the plane frame structure

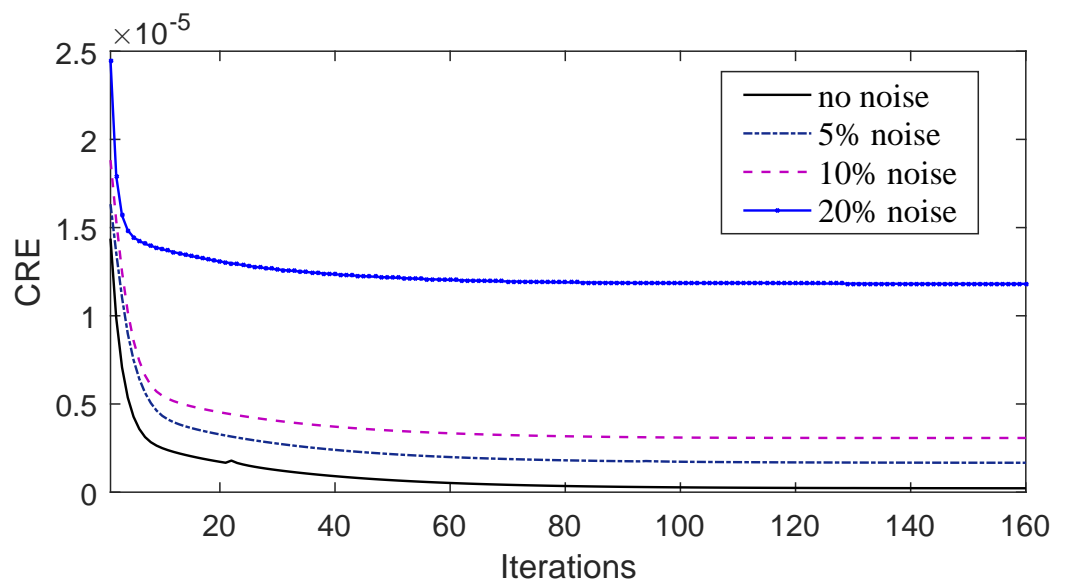

Figure $12 \mathrm{CRE}$ vs iterations for damage scenario 5 with different levels of noise 
Table 4 Damage scenarios for plane frame structure

\begin{tabular}{ccccc}
\hline $\begin{array}{c}\text { Damage scenario } \\
\text { scenario }\end{array}$ & $\begin{array}{c}\text { Damage location } \\
\text { (element set no.) }\end{array}$ & $\begin{array}{c}\text { Reduction in } \\
\text { elastic modulus (\%) }\end{array}$ & Noise (\%) & Measurement \\
\hline 1 & 3 & 10 & Nil & $\ddot{u}_{2}, \ddot{u}_{9}$ \\
2 & 3 & 20 & Nil & $\ddot{u}_{2}, \ddot{u}_{9}$ \\
3 & 3 & 40 & Nil & $\ddot{u}_{2}, \ddot{u}_{9}$ \\
4 & 3 & 10 & 10 & $\ddot{u}_{2}, \ddot{u}_{9}$ \\
5 & 3 & 20 & 10 & $\ddot{u}_{2}, \ddot{u}_{9}$ \\
6 & 3 & 40 & 10 & $\ddot{u}_{2}, \ddot{u}_{9}$ \\
7 & 4,5 & 10,5 & Nil & $\ddot{u}_{2}, \ddot{u}_{4}, \ddot{u}_{9}, \ddot{v}_{7}$ \\
8 & 4,5 & 20,25 & Nil & $\ddot{u}_{2}, \ddot{u}_{4}, \ddot{u}_{9}, \ddot{v}_{7}$ \\
9 & 4,5 & 50,50 & Nil & $\ddot{u}_{2}, \ddot{u}_{4}, \ddot{u}_{9}, \ddot{v}_{7}$ \\
10 & 4,5 & 10,5 & 10 & $\ddot{u}_{2}, \ddot{u}_{4}, \ddot{u}_{9}, \ddot{v}_{7}$ \\
11 & 4,5 & 20,25 & 10 & $\ddot{u}_{2}, \ddot{u}_{4}, \ddot{u}_{9}, \ddot{v}_{7}$ \\
12 & 4,5 & 50,50 & 10 & $\ddot{u}_{2}, \ddot{u}_{4}, \ddot{u}_{9}, \ddot{v}_{7}$ \\
\hline
\end{tabular}

Table 5 Frequency ranges for scenarios 1-12 of plane frame structure $(\mathrm{Hz})$

\begin{tabular}{ccccccc}
\hline Scenario & $1 \& 4$ & $2 \& 5$ & $3 \& 6$ & $7 \& 10$ & $8 \& 11$ & $9 \& 12$ \\
\hline Frequency ranges & $50-82$ & $50-82$ & $46-82$ & $46-74$ & $50-82$ & $50-82$ \\
$(4 \mathrm{~Hz}$ intervals $)$ & $143-147$ & $143-147$ & $139-143$ & $139-143$ & $139-143$ & $127-135$ \\
& $157-161$ & $153-157$ & $157-161$ & $157-161$ & $157-161$ & $149-161$ \\
& $169-185$ & $165-185$ & $169-181$ & $169-185$ & $169-185$ & $165-177$ \\
& $199-215$ & $195-219$ & $195-211$ & $199-219$ & $195-215$ & $187-203$ \\
& $230-262$ & $226-258$ & $226-258$ & $230-262$ & $226-258$ & $215-251$ \\
& $316-352$ & $316-352$ & $308-336$ & $316-352$ & $316-348$ & $300-316$ \\
$382-410$ & $378-394$ & $386-422$ & $382-410$ & $382-410$ & $378-402$ \\
\hline
\end{tabular}




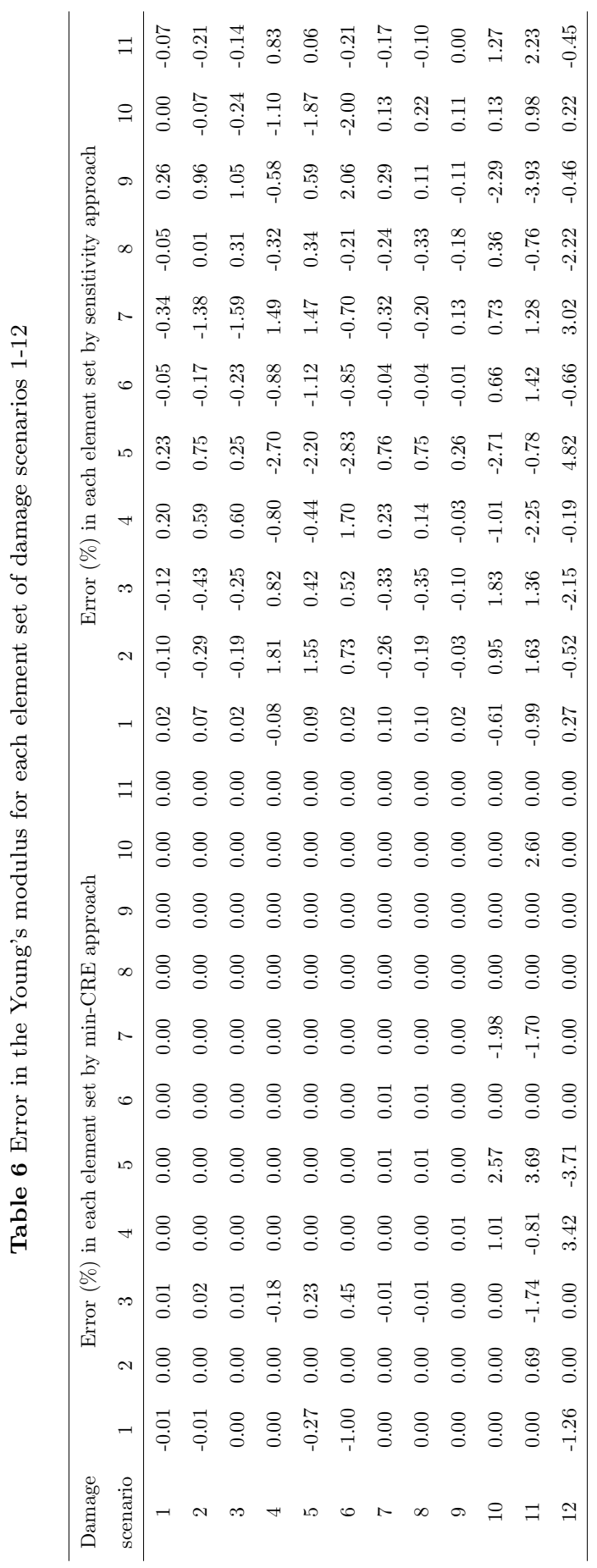




\subsection{A three-dimensional frame structure}

From the results of the identified stiffness reduction under the measurement noise and without the sparse regularization (Figure 15(b)), damage locations and extents became almost unidentifiable, while the sparse regularization substantially improves the accuracy and robustness of the identification procedure

${ }_{435}$ (Figure 15(a)). The proposed damage identification approach with sparsity is shown to be well applicable to the identification in 3D frames.

To show the convergence of the proposed spare-regularized min-CRE approach, filled contour plot of the damage parameters $\tilde{\boldsymbol{\theta}}$ was drawn in Figure 16. As it is seen, damages are identified after 35 steps, verifying the convergence 


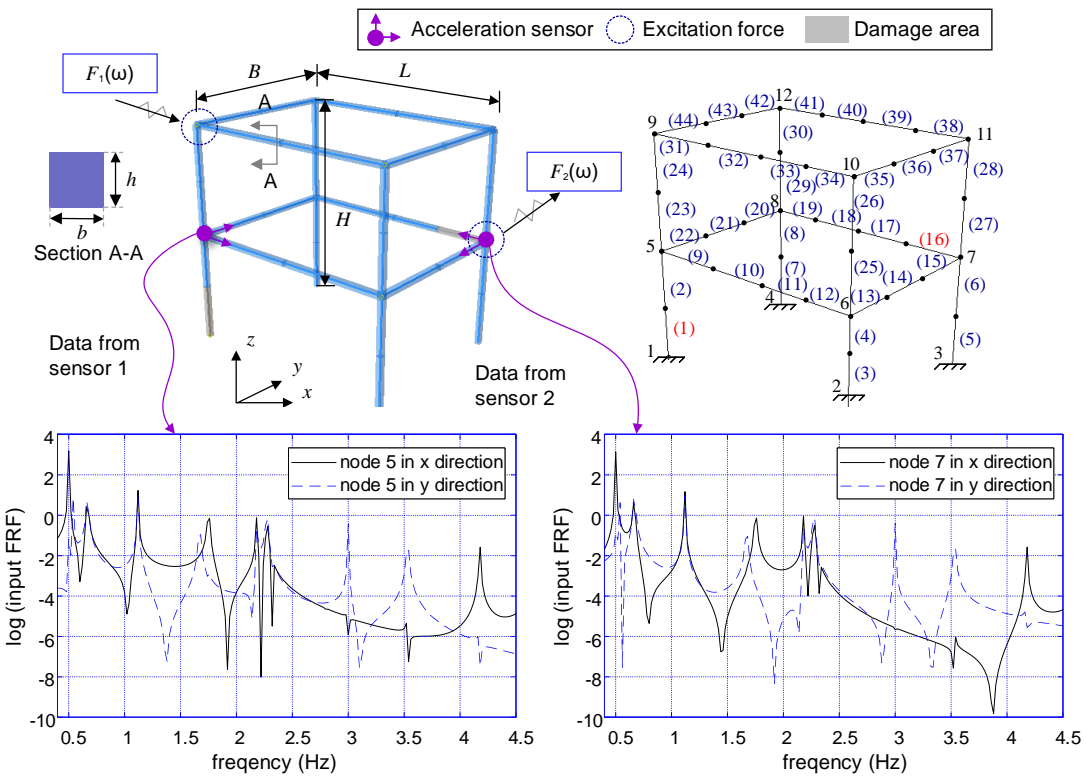

Figure 13 Schematic diagram showing the numerical structure and the directions of system output measurements and input excitations

of the present method. Algorithm without sparsity would encounter divergence or convergence difficulty with the same amount of input measurement data. This phenomenon is attributable to the fact that sparse regularization provides a flexible and parsimonious reconstructing process for high-dimensional model parameters from limited incomplete input data.

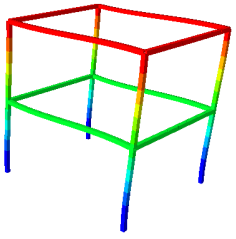

(a) $f=0.50 \mathrm{~Hz}$

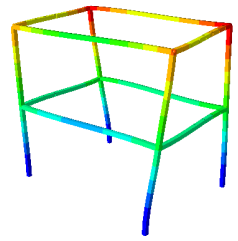

(b) $f=0.65 \mathrm{~Hz}$

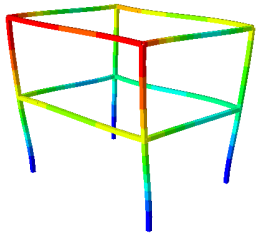

(c) $f=1.20 \mathrm{~Hz}$

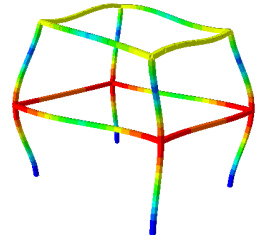

(d) $f=2.25 \mathrm{~Hz}$

Figure 14 Displacement fields for different frequency 

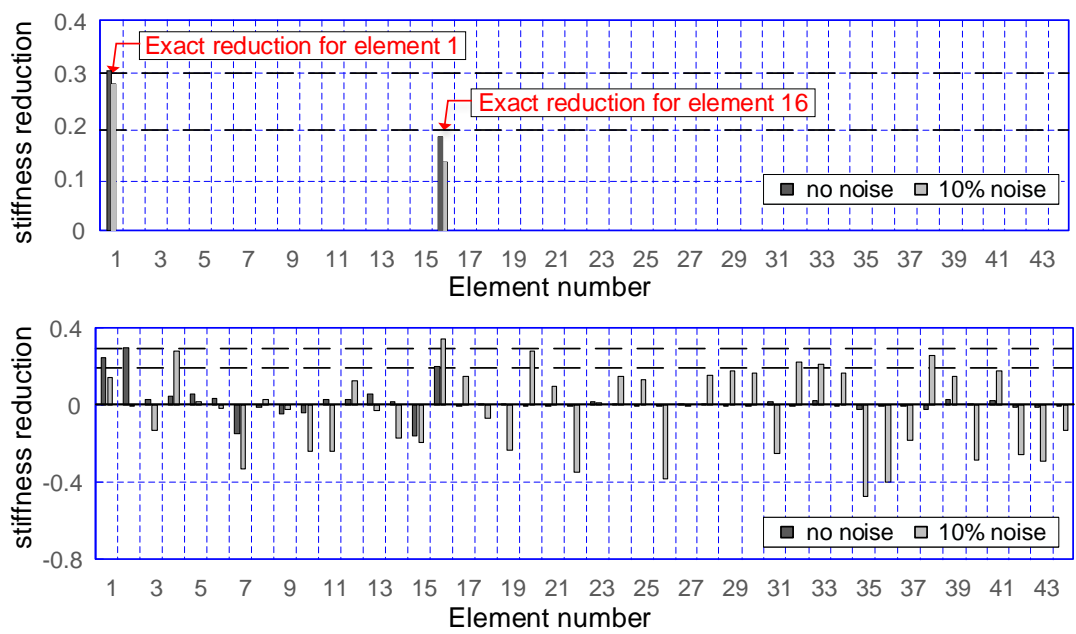

Figure 15 Stiffness reduction for each element: (a) the min-CRE approach with sparse regularization; (b) the min-CRE approach without sparse regularization

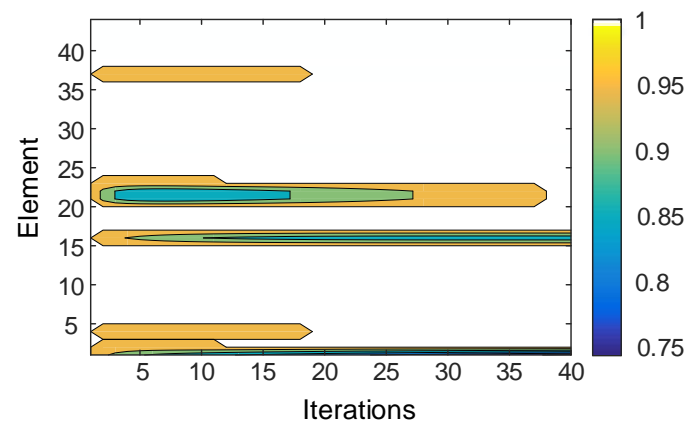

Figure 16 Contour plot of damage parameters for each element 


\section{Conclusions}

This research is focused on the damage identification approach with incompleted frequency response data using the minimum constitutive relation error (min-CRE) principle and the sparse regularization. The identification process is a nonlinear optimization problem and the corresponding objective function is set to measure the residual of the constitutive equations connecting the admissible stress field and the admissible displacement field. Numerical examples are conducted to verify the present approach and the results show that:

1. The present damage identification approach performs well in both damage localization and extent assessment for $2 \mathrm{D} / 3 \mathrm{D}$ frame structures with incomplete frequency response data.

2. The AM method could effectively tackle the nonlinear optimization problem.

3. The sparse regularization obviously improves the accuracy and robustness of the damage identification results.

4. The sparse regularization along with the CRE objective function is shown to be more accurate than the Tikhonov regularization along with the sensitivity identification approach.

5. The proposed threshold setting method could be used to determine the sparse regularization parameter automatically and efficiently.

6. The proposed approach is found to be much more efficient and less sensitive to the mass modeling errors than the sensitivity-based approaches (with the Tikhonov regularization or with the sparse regularization).

\section{APPENDIX}

\section{A.1. The displacement finite element}

The auxiliary local nodal displacements $\left(u_{x}^{i}, u_{y}^{i}, u_{z}^{i}, \theta_{x}^{i}, \theta_{y}^{i}, \theta_{z}^{i}\right)$ (3 translations and 3 rotation) at an arbitrary node $i$ are naturally selected to obtain the 
admissible displacement solution first. Thus, for an arbitrary element $e$ with two nodes $i, j$, the discretized displacement field $\left(w_{x}^{e}, w_{y}^{e}, w_{z}^{e}, \theta_{x}^{e}\right)$ as shown in Figure A.1 constructed by linear Lagrange interpolation for $\left(w_{x}^{e}, \theta_{x}^{e}\right)$ and cubic Hermit interpolation for $\left(w_{y}^{e}, w_{z}^{e}\right)$ based on the corresponding local nodal displacements $\left(u_{x}^{i}, u_{y}^{i}, u_{z}^{i}, \theta_{x}^{i}, \theta_{y}^{i}, \theta_{z}^{i}\right)$ and $\left(u_{x}^{j}, u_{y}^{j}, u_{z}^{j}, \theta_{x}^{j}, \theta_{y}^{j}, \theta_{z}^{j}\right)$, is expressed as

$$
\begin{gathered}
\left(\begin{array}{c}
w_{x}^{e} \\
w_{y}^{e} \\
w_{z}^{e} \\
\theta_{x}^{e}
\end{array}\right)=\Phi_{u}^{e} \tilde{\boldsymbol{u}}_{L}^{e}, \tilde{\boldsymbol{u}}_{L}^{e}:=\left[u_{x}^{i}, u_{y}^{i}, u_{z}^{i}, \theta_{x}^{i}, \theta_{y}^{i}, \theta_{z}^{i}, u_{x}^{j}, u_{y}^{j}, u_{z}^{j}, \theta_{x}^{j}, \theta_{y}^{j}, \theta_{z}^{j}\right]^{T} \\
\boldsymbol{\Phi}_{u}^{e}:=\left(\begin{array}{cccccccccccc}
N_{1} & 0 & 0 & 0 & 0 & 0 & N_{2} & 0 & 0 & 0 & 0 & 0 \\
0 & H_{1} & 0 & 0 & 0 & H_{2} & 0 & H_{3} & 0 & 0 & 0 & H_{4} \\
0 & 0 & H_{1} & 0 & -H_{2} & 0 & 0 & 0 & H_{3} & 0 & -H_{4} & 0 \\
0 & 0 & 0 & N_{1} & 0 & 0 & 0 & 0 & 0 & N_{2} & 0 & 0
\end{array}\right)
\end{gathered}
$$

where the polynomial interpolation functions $N_{1}, N_{2}, H_{1}, H_{2}, H_{3}, H_{4}$ are of the following forms,

$$
\begin{aligned}
& N_{1}(\xi)=\frac{1-\xi}{2}, N_{2}(\xi)=\frac{1+\xi}{2} \\
& \left\{\begin{array}{l}
H_{1}(\xi)=\frac{1}{2}(\xi+2)(\xi-1)^{2}, H_{2}(\xi)=\frac{1}{4}(\xi+1)(\xi-1)^{2} \frac{h^{e}}{2}, \\
H_{3}(\xi)=\frac{1}{2}(2-\xi)(\xi+1)^{2}, H_{2}(\xi)=\frac{1}{4}(\xi-1)(\xi+1)^{2} \frac{h^{e}}{2}, \\
\xi=\frac{2\left(s-s_{1}^{e}\right)}{h^{e}}-1
\end{array}\right.
\end{aligned}
$$

In order to perform the further assembling computation, the local nodal displacements $\tilde{\boldsymbol{u}}_{L}^{e}$ need to be expressed in a common coordinate system or the global coordinate system. To this end, the relationship between local nodal displacements $\tilde{\boldsymbol{u}}_{L}^{e}$ and global nodal displacements $\tilde{\boldsymbol{u}}^{e}$ in element $e$ is explored, pertaining to

$$
\tilde{\boldsymbol{u}}_{L}^{e}=\boldsymbol{T}_{u}^{e} \tilde{\boldsymbol{u}}^{e},
$$


where $\boldsymbol{T}_{u}^{e}$ is the transformation matrix of the following form

$$
\begin{gathered}
\boldsymbol{T}_{u}^{e}=\left(\begin{array}{cccc}
\boldsymbol{t} & \mathbf{0} & \mathbf{0} & \mathbf{0} \\
\mathbf{0} & \boldsymbol{t} & \mathbf{0} & \mathbf{0} \\
\mathbf{0} & \mathbf{0} & \boldsymbol{t} & \mathbf{0} \\
\mathbf{0} & \mathbf{0} & \mathbf{0} & \boldsymbol{t}
\end{array}\right) \\
\boldsymbol{t}=\left(\begin{array}{ccc}
\cos (x, \bar{x}) & \cos (x, \bar{y}) & \cos (x, \bar{z}) \\
\cos (y, \bar{x}) & \cos (y, \bar{y}) & \cos (y, \bar{z}) \\
\cos (z, \bar{x}) & \cos (z, \bar{y}) & \cos (z, \bar{z})
\end{array}\right)
\end{gathered}
$$

$\boldsymbol{t}$ is the Direction Cosine Matrix (DCM) between local coordinate system (Oxyz) and global coordinate system $(O \bar{x} \bar{y} \bar{z})$.

For brevity, the displacement finite element approximation is designated as

$$
\left(\begin{array}{c}
w_{x}^{e} \\
w_{y}^{e} \\
w_{z}^{e} \\
\theta_{x}^{e}
\end{array}\right)=\boldsymbol{N}_{1}^{e} \tilde{\boldsymbol{u}}^{e}, \boldsymbol{N}_{1}^{e}:=\boldsymbol{\Phi}_{u}^{e} \boldsymbol{T}_{u}^{e}
$$

\section{A.2. The force finite element}

In the force finite dimensional space, the discretized stress field $\left(N_{x}^{e}, M_{z}^{e}\right.$, $M_{y}^{e}, M_{x}^{e}$ ) (see Figure A.2) for an arbitary element $e$ should be constructed at first. Practically, this can be reached by setting

$$
\left(N_{x}^{e}, M_{z}^{e}, M_{y}^{e}, M_{x}^{e}\right)=\left(N_{x}^{0 e}, M_{z}^{0 e}, M_{y}^{0 e}, M_{x}^{0 e}\right)+\left(N_{x}^{p e}, M_{z}^{p e}, M_{y}^{p e}, M_{x}^{p e}\right)
$$

where $\left(N_{x}^{p e}, M_{z}^{p e}, M_{y}^{p e}, M_{x}^{p e}\right)$ is the inhomogeneous part caused by the concentrated loads on Neumann boundary $\partial \Omega_{f}$ and $\left(N_{x}^{0 e}, M_{z}^{0 e}, M_{y}^{0 e}, M_{x}^{0 e}\right)$ is the homogeneous part approximated by the following force element.

Auxiliary local nodal forces $\left(N_{x}^{i}, V_{y}^{i}, V_{z}^{i}, M_{x}^{i}, M_{y}^{i}, M_{z}^{i}\right)$ at an arbitrary node $i$ are used to construct the homogeneous discretized stress field $\left(N_{x}^{0}, M_{z}^{0}, M_{y}^{0}, M_{x}^{0}\right)$ 
initially; that is, for element $e,\left(N_{x}^{0 e}, M_{z}^{0 e}, M_{y}^{0 e}, M_{x}^{0 e}\right)$ are given by

$$
\begin{aligned}
\left(N_{x}^{0 e}, M_{z}^{0 e}, M_{y}^{0 e}, M_{x}^{0 e}\right)^{T}=\boldsymbol{\Phi}_{q}^{e} \tilde{\boldsymbol{q}}_{0 L}^{e}, \\
\tilde{\boldsymbol{q}}_{0 L}^{e}:=\left[N_{x}^{i}, V_{y}^{i}, V_{z}^{i}, M_{x}^{i}, M_{y}^{i}, M_{z}^{i}, N_{x}^{j}, V_{y}^{j}, V_{z}^{j}, M_{x}^{j}, M_{y}^{j}, M_{z}^{j}\right]^{T} \\
\boldsymbol{\Phi}_{q}^{e}:=\left(\begin{array}{cccccccccccc}
N_{1} & 0 & 0 & 0 & 0 & 0 & N_{2} & 0 & 0 & 0 & 0 & 0 \\
0 & -H_{2} & 0 & 0 & 0 & H_{1} & 0 & -H_{4} & 0 & 0 & 0 & H_{3} \\
0 & 0 & H_{2} & 0 & H_{1} & 0 & 0 & 0 & H_{4} & 0 & H_{3} & 0 \\
0 & 0 & 0 & N_{1} & 0 & 0 & 0 & 0 & 0 & N_{2} & 0 & 0
\end{array}\right)
\end{aligned}
$$

where $\boldsymbol{\Phi}_{q}^{e}$ is the shape function matrix. Having gained $\left(N_{x}^{0 e}, M_{z}^{0 e}, M_{y}^{0 e}, M_{x}^{0 e}\right)$ in equation (A-8) via the auxiliary local nodal forces, the next is to shift into the usage of global nodal forces. To this end, the transformation matrix for the force finite element pertains to be

$$
\boldsymbol{T}_{q}^{e}=\boldsymbol{H} \boldsymbol{T}_{u}^{e}
$$

where

$$
\boldsymbol{H}=\left(\begin{array}{cc}
-\boldsymbol{I}_{6 \times 6} & \mathbf{0} \\
\mathbf{0} & \boldsymbol{I}_{6 \times 6}
\end{array}\right)
$$

Hence, the relationship between local element nodal forces $\tilde{\boldsymbol{q}}_{0 L}^{e}$ and global element nodal forces $\tilde{\boldsymbol{q}}_{0}^{e}$ in terms of element $e$ is given by

$$
\tilde{\boldsymbol{q}}_{0 L}^{e}=\boldsymbol{T}_{q}^{e} \tilde{\boldsymbol{q}}_{0}^{e}
$$

Next, the formulation of nodal equilibriums is utilized to find independent global nodal force (DOFs) $\hat{\boldsymbol{q}}_{0}^{e}$. Element nodal forces $\tilde{\boldsymbol{q}}_{0}^{e i}=\left[F_{x}^{e i}, F_{y}^{e i}, F_{z}^{e i}, M_{x}^{e i}\right.$, $\left.M_{y}^{e i}, M_{z}^{e i}\right]^{T}$ of all $r$ beams as well as reaction forces $\boldsymbol{R}_{G}^{i}=\left[R_{x}^{i}, R_{y}^{i}, R_{z}^{i}, R M_{x}^{i}\right.$, $\left.R M_{y}^{i}, R M_{z}^{i}\right]^{T}$ adjacent to the objective node $i$ in the directions of all the degrees of freedom are algebraically summed up as shown in Figure A.3. This is carried out for all nodes. The equilibrium equation for each node $i$ is accordingly given by

$$
\sum_{e=1}^{r} \tilde{\boldsymbol{q}}_{0}^{e i}+\boldsymbol{R}_{G}^{i}=\mathbf{0}
$$


Based on this equilibrium equation, dependent nodal forces $\tilde{\boldsymbol{q}}_{0}^{r i}$ could be represented by independent global nodal forces $\hat{\boldsymbol{q}}_{0}^{e i}:=\left(\tilde{\boldsymbol{q}}_{0}^{e i}, e=1,2, \cdots, r-\right.$ $1, \boldsymbol{R}_{G}^{i}$ ) for the node $i$ in the sequel

$$
\boldsymbol{n} \hat{\boldsymbol{q}}_{0}^{e i}=-\sum_{e=1}^{r-1} \tilde{\boldsymbol{q}}_{0}^{e i}-\boldsymbol{R}_{G}^{i}=\tilde{\boldsymbol{q}}_{0}^{r i}
$$

where $\boldsymbol{n}$ stands for the linear combination of $\left\{\tilde{\boldsymbol{q}}_{0}^{e i}, e=1,2, \cdots, r-1\right\}$ and $\boldsymbol{R}_{G}^{i}$. It should be noticed that homogeneous Neumann boundary conditions should be enforced on reaction forces $\boldsymbol{R}_{G}^{i}$, setting corresponding forces to be zero for different types of supports. Thus, one can obtain for an element $e$ with an ending node $i$ that

$$
\left[F_{x}^{e i}, F_{y}^{e i}, F_{z}^{e i}, M_{x}^{e i}, M_{y}^{e i}, M_{z}^{e i}\right]^{T}=\boldsymbol{L}_{i}^{e} \hat{\boldsymbol{q}}_{0}^{e i}
$$

where

$$
\begin{aligned}
\boldsymbol{L}_{i}^{e}(3 e-2: 3 e, 3 e-2: 3 e) & =\boldsymbol{I}_{3 \times 3} \text { for } e=1,2, \cdots, r-1 \\
\boldsymbol{L}_{i}^{e} & =\boldsymbol{n} \text { for } e=r
\end{aligned}
$$

Integrating Equations (A-8),(A-11) and (A-14), the force finite element approximation of $\left(\hat{N}_{h}^{e}, \hat{M}_{h}^{e}\right)$ is established as

$$
\begin{aligned}
& \left(\begin{array}{c}
N_{x}^{0 e} \\
M_{z}^{0 e} \\
M_{y}^{0 e} \\
M_{x}^{0 e}
\end{array}\right)=\boldsymbol{\Psi}_{q}^{e} \hat{\boldsymbol{q}}_{0}^{e}=\boldsymbol{N}_{2}^{e} \tilde{\boldsymbol{q}}_{0}^{e} \\
& \boldsymbol{\Psi}_{q}^{e}:=\boldsymbol{N}_{2}^{e}\left(\begin{array}{cc}
\boldsymbol{L}_{i}^{e} & \mathbf{0} \\
\mathbf{0} & \boldsymbol{L}_{i}^{e}
\end{array}\right)=\boldsymbol{\Phi}_{q}^{e} \boldsymbol{T}_{q}^{e}\left(\begin{array}{cc}
\boldsymbol{L}_{i}^{e} & \mathbf{0} \\
\mathbf{0} & \boldsymbol{L}_{i}^{e}
\end{array}\right) \\
& \tilde{\boldsymbol{q}}_{0}^{e}=\left(\begin{array}{cc}
\boldsymbol{L}_{i}^{e} & \mathbf{0} \\
\mathbf{0} & \boldsymbol{L}_{i}^{e}
\end{array}\right) \hat{\boldsymbol{q}}_{0}^{e}=\left(\begin{array}{cc}
\boldsymbol{L}_{i}^{e} & \mathbf{0} \\
\mathbf{0} & \boldsymbol{L}_{i}^{e}
\end{array}\right)\left(\begin{array}{c}
\hat{\boldsymbol{q}}_{0}^{i} \\
\hat{\boldsymbol{q}}_{0}^{j}
\end{array}\right)
\end{aligned}
$$

Turning to the particular part $\left(N_{x}^{p e}, M_{z}^{p e}, M_{y}^{p e}, M_{x}^{p e}\right)$, the external concentrated loads are directly involved. Assume that the concentrated loads $\tilde{\boldsymbol{q}}_{p}^{e}:=$ $\left[F_{p x}, F_{p y}, F_{p z}, M_{p x}, M_{p y}, M_{p z}\right]^{T}$ are enforced on the left-hand side $k=1$ (or right-hand side $k=2)$ of the element $e$. Under this circumstance, $\left(N_{x}^{p e}, M_{z}^{p e}\right.$, 


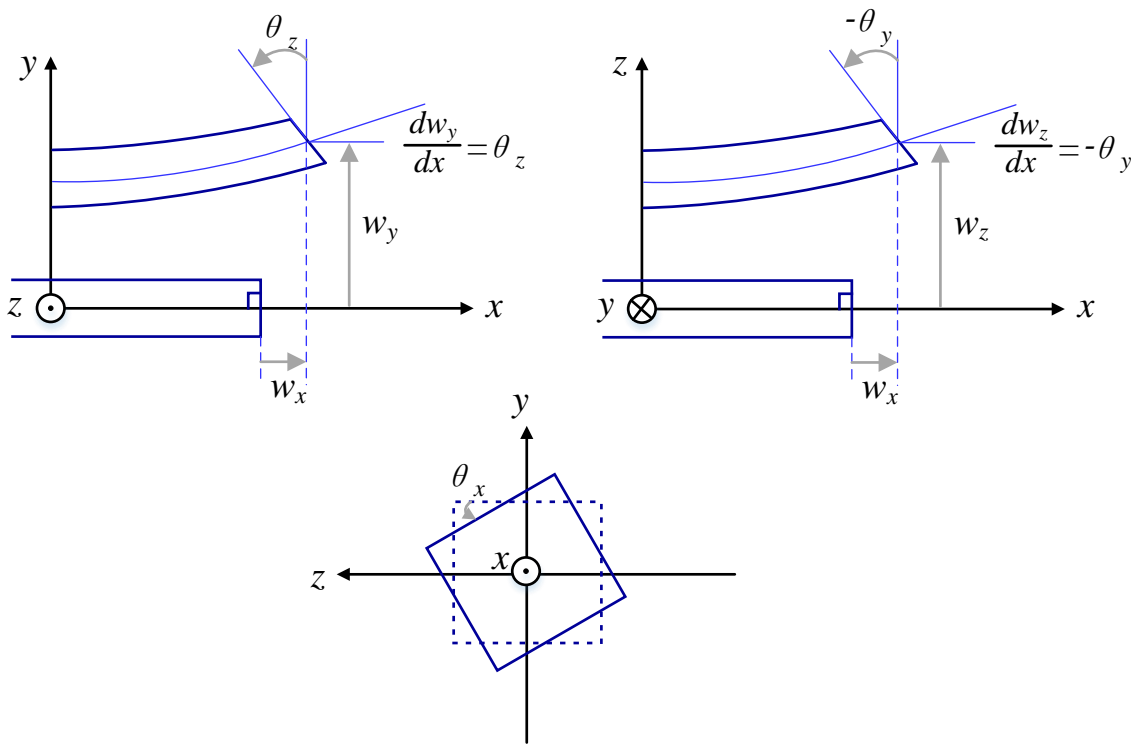

Figure A. .1 Basics of a displacement finite element $e$

$\left.M_{y}^{p e}, M_{x}^{p e}\right)$ could be obtained by the interpolation matrix defined in equation (A-1) and transformation matrix defined in equation (A-9) as follows

$$
\left(\begin{array}{c}
N_{x}^{p e} \\
M_{z}^{p e} \\
M_{y}^{p e} \\
M_{x}^{p e}
\end{array}\right)=N_{2}^{e} \tilde{\boldsymbol{q}}_{p}^{e}
$$

where $\tilde{\boldsymbol{q}}_{p}$ collects all concentrated loads $\left(F_{p x}, F_{p y}, F_{p z}, M_{p x}, M_{p y}, M_{p z}\right)$.

Finally, substituting Equation (A-16) and (A-17) into Equation (A-7) and simplifying, one can get

$$
\left(\begin{array}{c}
N_{x}^{e} \\
M_{z}^{e} \\
M_{y}^{e} \\
M_{x}^{e}
\end{array}\right)=\boldsymbol{N}_{2}^{e} \tilde{\boldsymbol{q}}_{0}^{e}+\boldsymbol{N}_{2}^{e} \tilde{\boldsymbol{q}}_{p}^{e}
$$




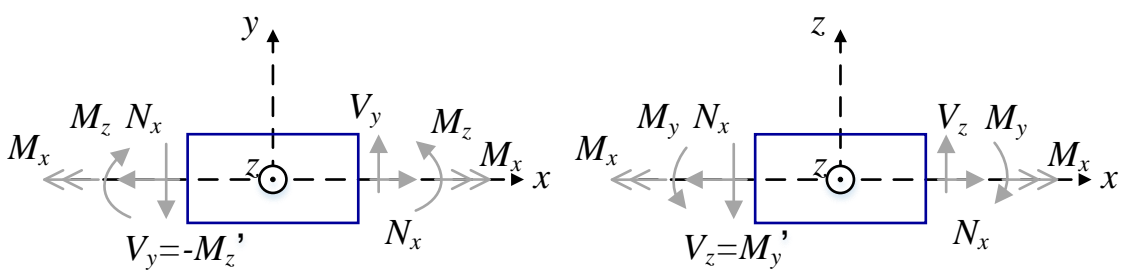

Figure A. .2 Basics of a force finite element $e$
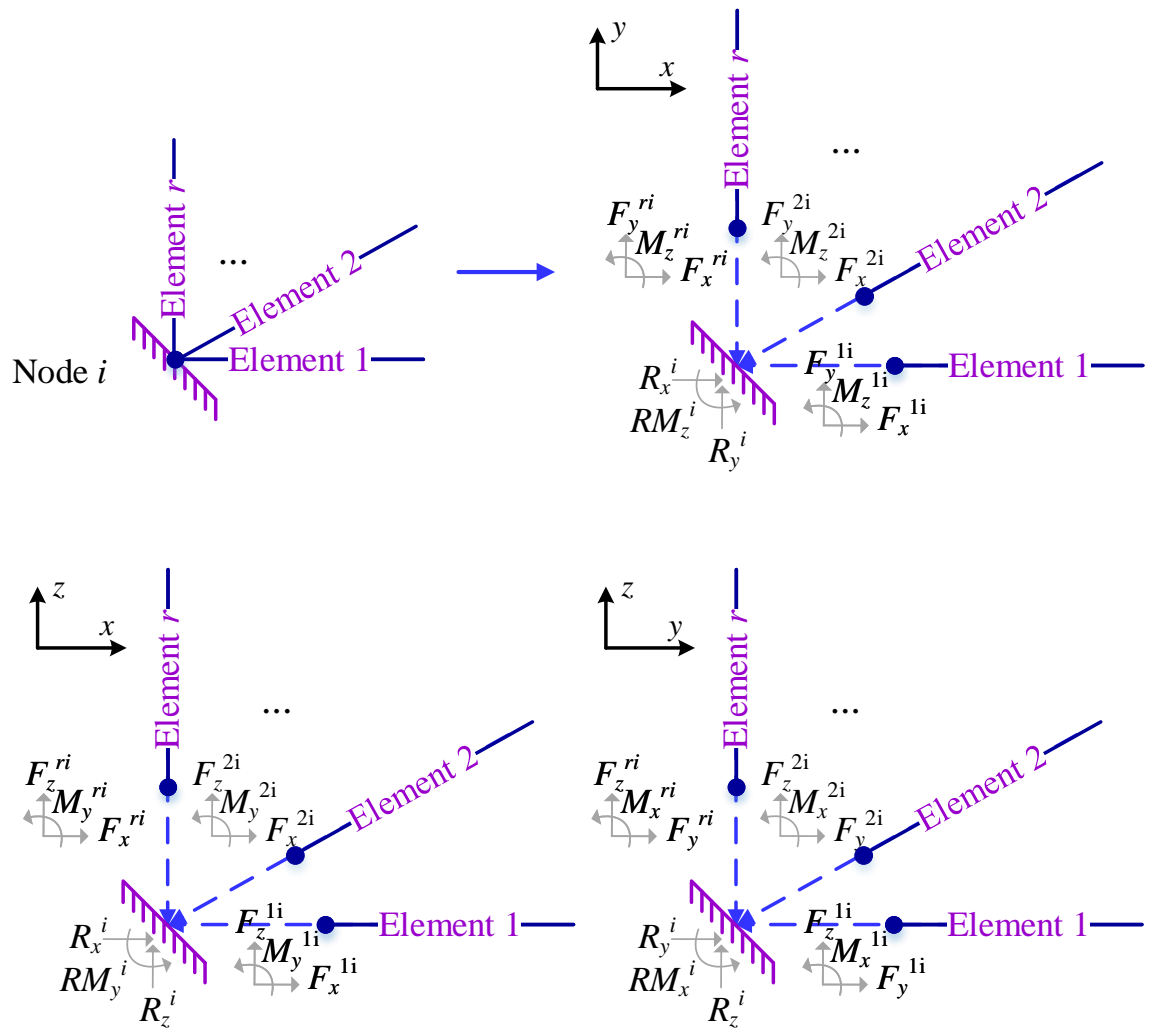

Figure A. .3 Equilibrium at a node $i$ 


\section{Acknowledgement}

The present research was performed under the support of the MEXT scholarship of Japan.

\section{References}

\section{References}

[1] P. G. Bakir, E. Reynders, G. De Roeck, Sensitivity-based finite element model updating using constrained optimization with a trust region algorithm, Journal of Sound and Vibration 305 (1-2) (2007) 211-225.

[2] Z. Zheng, Z. Lu, W. Chen, J. Liu, Structural damage identification based on power spectral density sensitivity analysis of dynamic responses, Computers \& Structures 146 (2015) 176-184.

[3] Z. Lu, S. Law, Features of dynamic response sensitivity and its application in damage detection, Journal of Sound and Vibration 303 (1-2) (2007) 305329.

[4] G. Liu, S. Chen, A novel technique for inverse identification of distributed stiffness factor in structures, Journal of Sound and Vibration 254 (5) (2002) 823-835.

[5] O. Begambre, J. E. Laier, A hybrid particle swarm optimization-simplex algorithm (PSOS) for structural damage identification, Advances in Engineering Software 40 (9) (2009) 883-891.

[6] A. Hera, Z. Hou, Application of wavelet approach for asce structural health monitoring benchmark studies, Journal of Engineering Mechanics 130 (1) (2004) 96-104.

[7] N. Roveri, A. Carcaterra, Damage detection in structures under traveling loads by hilbert-huang transform, Mechanical Systems and Signal Processing 28 (2012) 128-144. 
[8] B. Jaishi, W.-X. Ren, Damage detection by finite element model updating using modal flexibility residual, Journal of Sound and Vibration 290 (1-2) (2006) 369-387.

[9] Z.-R. Lu, L. Wang, An enhanced response sensitivity approach for structural damage identification: convergence and performance, International journal for numerical methods in engineering 111 (13) (2017) 1231-1251.

[10] J. Cunha, S. Cogan, C. Berthod, Application of genetic algorithms for the identification of elastic constants of composite materials from dynamic tests, International Journal for Numerical Methods in Engineering 45 (7) (1999) 891-900.

[11] G. Liu, S. Chen, Flaw detection in sandwich plates based on time-harmonic response using genetic algorithm, Computer Methods in Applied Mechanics and Engineering 190 (42) (2001) 5505-5514.

[12] H.-P. Chen, T. S. Maung, Regularised finite element model updating using measured incomplete modal data, Journal of Sound and Vibration 333 (21) (2014) 5566-5582.

[13] M. Kaouk, D. C. Zimmerman, Structural damage assessment using a generalized minimum rank perturbation theory, AIAA Journal 32 (4) (1994) 836-842.

[14] I. Ojalvo, D. Pilon, Diagnostics for geometrically locating structural math model errors from modal test data, in: 29th Structures, Structural Dynamics and Materials Conference, 1988, p. 2358.

[15] J. Guo, I. Takewaki, Minimum constitutive relation error based static identification of beams using force method, Journal of Physics: Conference Series 842 (1) (2017) 012026.

[16] P. Feissel, O. Allix, Modified constitutive relation error identification strategy for transient dynamics with corrupted data: The elastic case, Computer Methods in Applied Mechanics and Engineering 196 (13) (2007) 1968-1983. 
[17] B. Blaysat, E. Florentin, G. Lubineau, A. Moussawi, A dissipation gap method for full-field measurement-based identification of elasto-plastic material parameters, International Journal for Numerical Methods in Engineering 91 (7) (2012) 685-704.

[18] M. Bonnet, A. Constantinescu, Inverse problems in elasticity, Inverse Problems 21 (2) (2005) R1.

[19] B. Banerjee, T. F. Walsh, W. Aquino, M. Bonnet, Large scale parameter estimation problems in frequency-domain elastodynamics using an error in constitutive equation functional, Computer Methods in Applied Mechanics and Engineering 253 (2013) 60-72.

[20] B. Titurus, M. Friswell, Regularization in model updating, International Journal for Numerical Methods in Engineering 75 (4) (2008) 440-478.

[21] C. Zhang, J.-Z. Huang, G.-Q. Song, L. Chen, Structural damage identification by extended kalman filter with $\ell_{1}$-norm regularization scheme, Structural Control and Health Monitoring 24 (11).

[22] E. M. Hernandez, Identification of isolated structural damage from incomplete spectrum information using 11-norm minimization, Mechanical Systems and Signal Processing 46 (1) (2014) 59-69.

[23] Y. Wang, H. Hao, Damage identification scheme based on compressive sensing, Journal of Computing in Civil Engineering-ASCE 29 (2) (2013) 04014037.

[24] V. A. Morozov, Methods for solving incorrectly posed problems, Springer Science \& Business Media, 2012.

[25] G. H. Golub, M. Heath, G. Wahba, Generalized cross-validation as a method for choosing a good ridge parameter, Technometrics 21 (2) (1979) $215-223$. 
[26] P. C. Hansen, Analysis of discrete ill-posed problems by means of the lcurve, SIAM review 34 (4) (1992) 561-580.

[27] D. Mascareñas, A. Cattaneo, J. Theiler, C. Farrar, Compressed sensing techniques for detecting damage in structures, Structural Health Monitoring 12 (4) (2013) 325-338.

[28] Y. Yang, S. Nagarajaiah, Structural damage identification via a combination of blind feature extraction and sparse representation classification, Mechanical Systems and Signal Processing 45 (1) (2014) 1-23.

[29] R. Hou, Y. Xia, Y. Bao, X. Zhou, Selection of regularization parameter for 11-regularized damage detection, Journal of Sound and Vibration 423 (2018) 141-160.

[30] D.-H. Lee, W.-S. Hwang, Parametric optimization of complex systems using a multi-domain FRF-based substructuring method, Computers \& structures 81 (22-23) (2003) 2249-2257.

[31] F. Vestroni, D. Capecchi, Damage detection in beam structures based on frequency measurements, Journal of Engineering Mechanics 126 (7) (2000) 761-768.

[32] A. Esfandiari, F. Bakhtiari-Nejad, A. Rahai, M. Sanayei, Structural model updating using frequency response function and quasi-linear sensitivity equation, Journal of Sound and Vibration 326 (3) (2009) 557-573.

[33] P. Ladeveze, D. Leguillon, Error estimate procedure in the finite element method and applications, SIAM Journal on Numerical Analysis 20 (3) (1983) 485-509.

580

[34] D. Barthe, A. Deraemaeker, P. Ladevèze, S. Le Loch, Validation and updating of industrial models based on the constitutive relation error, AIAA journal $42(7)$. 
[35] A. Beck, On the convergence of alternating minimization for convex programming with applications to iteratively reweighted least squares and decomposition schemes, SIAM Journal on Optimization 25 (1) (2015) 185209.

[36] E. J. Candes, M. B. Wakin, S. P. Boyd, Enhancing sparsity by reweighted $\ell_{1}$ minimization, Journal of Fourier Analysis and Applications 14 (5) (2008) 877-905.

[37] M. Schmidt, Least squares optimization with $L_{1}$-norm regularization, CS542B Project Report (2005) 14-18.

[38] M. Y. Park, T. Hastie, $L_{1}$-regularization path algorithm for generalized linear models, Journal of the Royal Statistical Society: Series B (Statistical Methodology) 69 (4) (2007) 659-677.

[39] J. E. Mottershead, M. Link, M. I. Friswell, The sensitivity method in finite element model updating: a tutorial, Mechanical systems and signal processing 25 (7) (2011) 2275-2296.

[40] Z.-R. Lu, J. Zhou, L. Wang, On choice and effect of weight matrix for response sensitivity-based damage identification with measurement and model errors, Mechanical Systems and Signal Processing 114 (2019) 1-24.

[41] J. A. Dos Santos, C. M. Soares, C. M. Soares, N. Maia, Structural damage identification in laminated structures using FRF data, Composite Structures 67 (2) (2005) 239-249.

[42] J. Friedman, T. Hastie, R. Tibshirani, The elements of statistical learning, Vol. 1, Springer series in statistics New York, 2001. 